Physical Processes Controlling Dark Current Emission and Resulting Breakdown in Linacs by I. Konkashbaev and A. Hassanein

Energy Technology Division Energy Technology Division Energy Technology Division Energy Technology Division Energy Technology Division Energy Technology Division Energy Technology Division Energy Technology Division Energy Technology Division Energy Technology Division Energy Technology Division Energy Technology Division Energy Technology Division Energy Technology Division Energy Technology Division Energy Technology Division Energy Technology Division Energy Technology Division Energy Technology Division Energy Technology Division

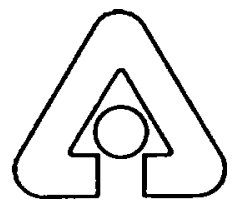

Argonne National Laboratory, Argonne, Illinois 60439 operated by The University of Chicago for the United States Department of Energy under Contract W-31-109-Eng-38

Energy Technology Division Energy Technology Division Energy Technology Division Energy Technology Division Energy Technology Division Energy Technology Division Energy Technology Division Energy Technology Division 
Argonne National Laboratory, a U.S. Department of Energy Office of Science Laboratory, is operated by The University of Chicago under contract W-31-109-Eng-38.

DISCLAIMER
This report was prepared as an account of work sponsored by an
agency of the United States Government. Neither the United States
Government nor any agency thereof, nor The University of Chicago,
nor any of their employees or officers, makes any warranty, express or
implied, or assumes any legal liability or responsibility for the
accuracy, completeness, or usefulness of any information, apparatus,
product, or process disclosed, or represents that its use would not
infringe privately owned rights. Reference herein to any specific
commercial product, process, or service by trade name, trademark,
manufacturer, or otherwise, does not necessarily constitute or imply
its endorsement, recommendation, or favoring by the United States
Government or any agency thereof. The views and opinions of
document authors expressed herein do not necessarily state or reflect
those of the United States Government or any agency thereof.

Available electronically at http://www.doe.gov/bridge

Available for a processing fee to U.S. Department of Energy and its contractors, in paper, from:

U.S. Department of Energy

Office of Scientific and Technical Information

P.O. Box 62

Oak Ridge, TN 37831-0062

phone: (865) 576-8401

fax: (865) 576-5728

email: reports@adonis.osti.gov 


\author{
Argonne National Laboratory \\ 9700 South Cass Ave \\ Argonne, IL 60439, USA
}

\title{
PHYSICAL PROCESSES CONTROLLING DARK CURRENT EMISSION AND RESULTING BREAKDOWN IN LINACS
}

By

I. Konkashbaev and A. Hassanein

Energy Technology Division

December 2003

\begin{abstract}
The submitted manuscript has been created by the University of Chicago as Operator of Argonne National Laboratory ("Argonne") under Contract No. W-31-109ENG-38 with the U.S. Department of Energy. The U.S. Government retains for itself, and others acting on its behalf, a paid-up, nonexclusive, irrevocable worldwide license in said article to reproduce, prepare derivative works, distribute copies to the public, and perform publicly and display publicly by or on behalf of the Government.
\end{abstract}




\section{CONTENTS}

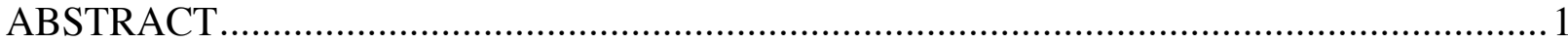

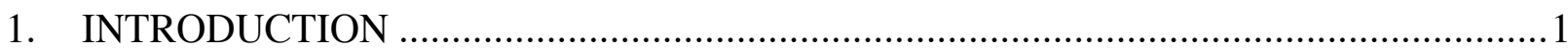

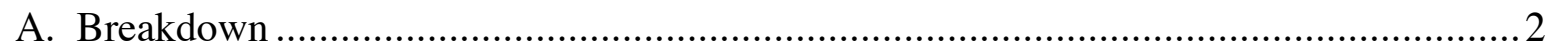

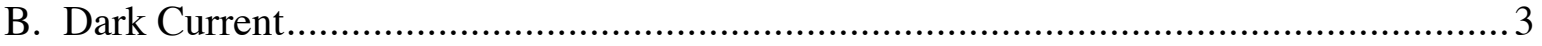

2. RADIO FREQUENCY (RF) BREAKDOWN ........................................................ 3

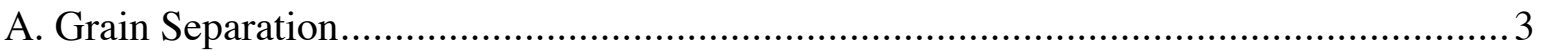

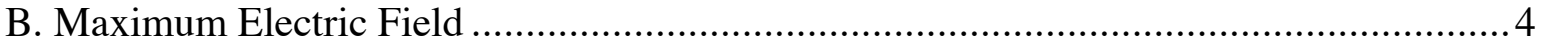

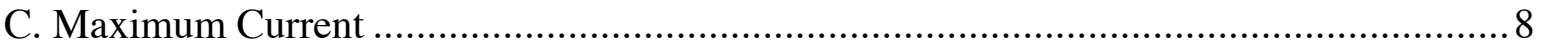

D. Minimum Size of Grains .............................................................................. 8

E. Maximum Number of Separating Grains ................................................... 8

F. Fowler-Nordheim Field Emission Current............................................................ 9

G. Child-Langmuir Emission Current ............................................................ 11

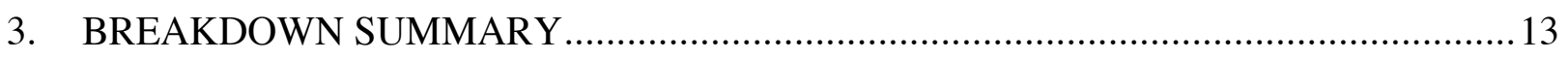

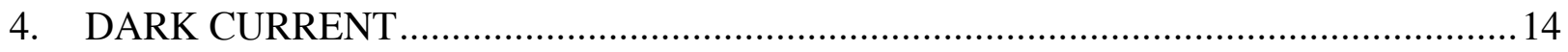

A. Stopping Power of the Relativistic Electrons and Secondary Emissions .................... 15

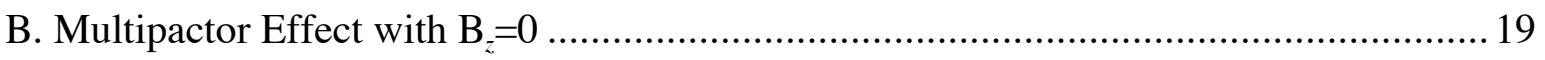

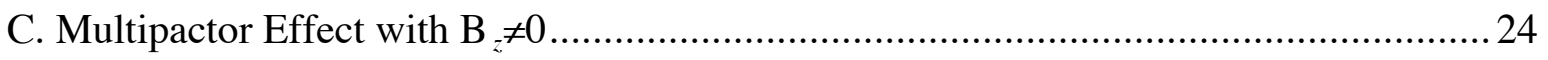

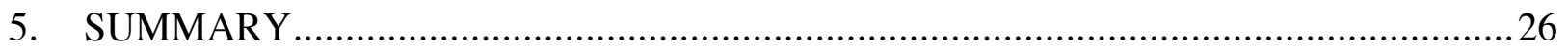

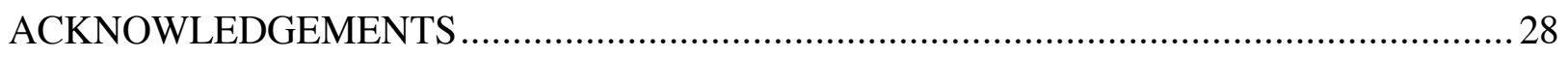

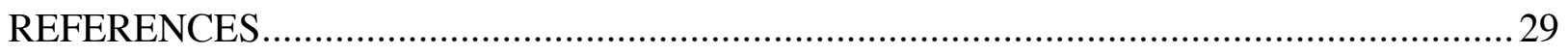




\section{FIGURES}

Fig. 1. Schematic of separation of grain from body by electric tension............................. 4

Fig. 2. Schematic of grain-by-grain separation ...........................................................

Fig. 3. Equipotential lines and electric fields for hemicylinder ................................... 5

Fig. 4. Streamline and potential near single cylinder .................................................. 6

Fig 5. Field enhancement as function of grain size ................................................... 7

Fig. 6. Destruction limit as function of grain size........................................................ 7

Fig. 7. Fowler-Nordheim field emission current.................................................. 9

Fig. 8. Dependence of the field emission on the spot size ........................................ 10

Fig. 9. Potential of spherical capacity with and without space charge............................... 12

Fig. 10. Breakdown threshold as function of frequency .......................................... 14

Fig. 11. Effect of radiation fluxes through electrode on electric field magnitude ................... 14

Fig. 12. Energetic spectrum of the secondary electrons .......................................... 15

Fig. 13. Electric field of stopped and moving charge ................................................ 17

Fig. 14a. Electron ejected at $\mathrm{r}=0$ and reached the opposite electrode ................................ 20

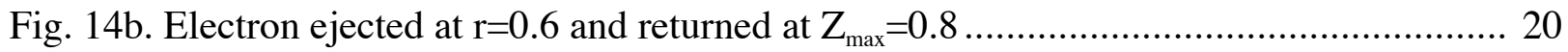

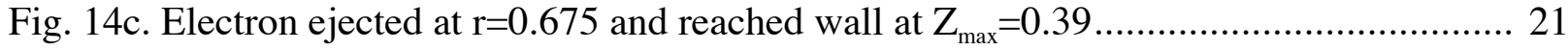

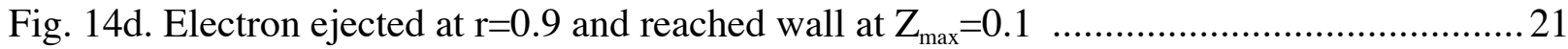

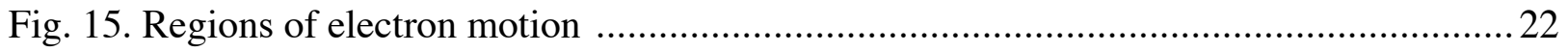

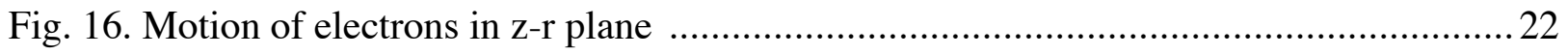

Fig. 17. Electron motion between walls at $\square=1$........................................................ 23

Fig. 18. Chain process of secondary electron. Points mean energy when electron strikes opposite

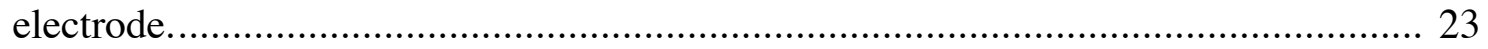

Fig. 19. Electron dynamics without guide magnetic field .......................................... 24

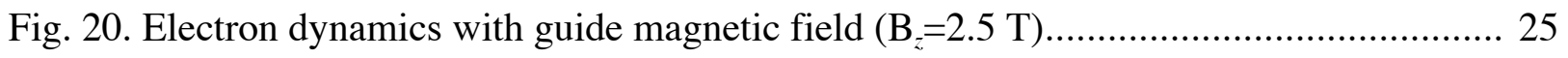

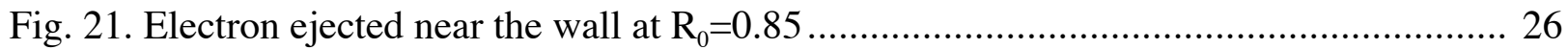

\section{TABLES}

Table 1. Parameters for current source spots ............................................................ 9

Table 2. Magnetic pressure and corresponding temperature .......................................... 10

Table 3. Spot parameters according to the Child-Langmuir current ..................................... 13 


\title{
PHYSICAL PROCESSES CONTROLLING DARK CURRENT EMISSION AND RESULTING BREAKDOWN IN LINACS
}

\author{
I. Konkashbaev and A. Hassanein \\ Argonne National Laboratory
}

\begin{abstract}
Higher accelerating gradients are required for future high-power devices such as the $\mathrm{TeV}$ electron linear collider and muon-muon collider. A limit in the maximum field gradient is imposed by the rf dark current and the resulting electrical breakdown. Two critical questions need to be answered: What causes high dark current and breakdown? Can the breakdown threshold be increased? These problems have been the focus of significant experimental and theoretical investigations for over a century. The purpose of this report is to shed some light on the possible mechanisms that limit the field gradient in linacs with and without guiding magnetic fields.

The idea of enhanced field emission due to separation of grains from a material surface by the electric field tension acting normally to the surface is discussed. The electric force can be high enough to overcome the binding energy of grains in the material. Thus, small-size separated grains result in enhanced field emission. The stopping power of relativistic and ultra-relativistic energies of ejected electrons at high electric field results in more production of secondary electrons. At high energy, $\mathrm{E}>>\mathrm{mc}^{2}$, the stopping power of relativistic and ultra-relativistic electrons becomes similar to the stopping power of ions with the same energy but much less velocity. It results in more production of the secondary electrons.

The strong influence of a guide magnetic field results in more electrons reaching the opposite electrode due to straightening of electron trajectory and, consequently, more production of the secondary electrons. For a more accurate understanding of these complicated issues, detailed experimental and theoretical investigations are required.
\end{abstract}

\section{INTRODUCTION}

Higher accelerating gradients are required for future high-power devices such as the $\mathrm{TeV}$ electron linear collider and muon-muon collider. A limit in the maximum field gradient (electric field $\mathrm{E}=-\square \mathrm{U}$, where $\mathrm{U}$ is the field potential) is imposed by the rf dark current following electrical breakdown. The focal point of this report is the possible mechanisms limiting the field gradient in linacs with and without a guide magnetic field.

Two critical questions are: What causes high dark current and breakdown? Can the breakdown threshold be increased? These problems have been the focus of experimental and theoretical investigations for over a century. Presently, how to resolve the problem of $\mathrm{rf}$ dark current and breakdown remains unclear. (An encouraging review of experimental and theoretical investigations completed through the year 2002 is found in [1].) The results of our investigation into these problems are summarized in the following report. 


\section{A. Breakdown}

In 1947, Trump and Van de Graaf proposed an electrode-ion exchange model to explain vacuum breakdown [2]. This theory describes breakdown to be the result of a regenerative process. According to the theory, a single electron emitted from the cathode causes the ejection of $A$ positive ions and $C$ photons when it strikes the anode. Then, if a positive ion produces $B$ secondary electrons and the one photon produces $D$ secondary electrons at the cathode, a runaway phenomenon is established when $A B+C D \geq 1$. The authors of this hypothesis found that the secondary emission factors were too small for the development of a cumulative process [3].

High-frequency rf breakdown at relatively low voltages is a single surface phenomenon. The field enhancement factor is used to match experimental data to theory. This factor takes into account a localized field enhancement due to microprotrusions or surface irregularities with $\mathrm{E}_{0}$ (the electric field at the electrode surface) and $E_{\text {act }}$ (the electric field necessary to satisfy the Fowler-Nordheim field emission formula):

$$
\mathrm{b}_{\mathrm{E}}=\frac{\mathrm{E}_{\text {act }}}{\mathrm{E}_{0}}
$$

The breakdown can be determined by electrode explosive emission [4], but it is necessary to find mechanisms that relate to current strong enough to form an ionized vapor cloud as a source of unlimited emission.

Multipactor discharge occurs if secondary emission yield exceeds unity, and the synchrony condition is satisfied. Usually the multipactor effect is low at relatively low electric field because the velocity of emitted secondary electrons is less than the speed of light c. The multipactor in superconductive cavities has been reduced or eliminated by using elliptical or spherical geometries.

Surface processing decreases the field enhancement factor, but there are limits to the surface improvement following treatment. Bohne et al. [5] found no improvement in the breakdown threshold for a surface finish below $1 \square \mathrm{m}$. A low secondary-emission coating on copper was tested. The results show reduced field emission until breakdown, after which the magnitude of the field emission increases to levels consistent with that of bare metals. Three predominant stages have been observed. Because the threshold for field emission occurs at $\mathrm{E}_{0}=80 \mathrm{MeV} / \mathrm{m}$, the first stage ranges from $\mathrm{E}_{0}=0-80 \mathrm{MeV} / \mathrm{m}$, where no X-ray is detected. The second stage occurs at $\mathrm{E}_{0}=80-190 \mathrm{MeV} / \mathrm{m}$, where the X-ray intensity level increases monotonically along the pre-breakdown curve. The X-ray intensity measurements are reproducible as long as the field gradients are below $\mathrm{E}_{0}=190 \mathrm{MeV} / \mathrm{m}$. The third stage is where irreversible damage (postbreakdown) occurs. Experiments with single-crystal materials produce similar results. Singlecrystal materials eliminate the concern about trapped gases and impurities on the electrode surface, such as microprotrusions and grain boundaries. Experiments with single-crystal copper have shown a field-emission current reduction of only 50\% compared with standard OFE copper.

There are a number of theoretical speculations on the nature of the breakdown, but the experimental breakdown limits were found to be lower that the theoretical calculations for all breakdown theories. The so-called Kilpatrick's [6] criterion summarizes experimental results: 


$$
f(M H z)=\square \cdot 1.643 E^{2} \exp \left(\square \frac{8.5}{E}\right), E\left(\frac{M e V}{m}\right)
$$

where the coefficient $\square$ depends on element charge, Z; atomic weight, A; and surface processing. This equation indicates that something like particle motion influences the breakdown field.

\section{B. Dark Current}

The problem of the dark current is still unsolved. The dark current dependence on $\mathrm{E}_{0}$ also has a threshold character. Matsumoto [7] found that the field emission current increased exponentially with structure length at relatively low accelerating gradients $(<60 \mathrm{MeV} / \mathrm{m})$ and increased linearly at higher accelerating gradients. At low accelerating gradients, the dark current increases as $\square^{\mathrm{N}}$, where $\mathrm{N}$ is the number of cells in the structure, and $\square$ is a constant. It was explained that field emission is a multiplication process, where emitted electrons from upstream cells are multiplied in the successive cells downstream. For such a multiplication effect to exist, the secondary emission coefficient $\square$ should exceed 1:

$$
\square=n_{e, \text { primary }} / n_{e, \text { secondary }} \geq 1
$$

A significant amount of experimental and calculated data have been obtained at low energy of incoming electrons $(\mathrm{E}<1 \mathrm{MeV})$, but interactions at higher energies have been studied much less.

\section{RADIO FREQUENCY (RF) BREAKDOWN}

As high-frequency rf breakdown is a single surface phenomenon, it is necessary to regard field emission of a surface with inhomogeneities such as microprotrusions having size comparable with grains, because conditioning can smooth the surface to grain-size irregularities. Let us regard the simplest case of a single-cavity (linac) cylindrical form with length $L$ and radius $R$ determined by frequency $\mathrm{f}$ and corresponding wavelength $\square$. For mode $\mathrm{TM}_{010} L=\square / 2$ and $R=2.405 \mathrm{~L}$. We consider here the ultimate case of the perfect surface (after fine conditioning) when the surface contains no absorbed gases or mechanical imperfections. The electrode would be polycrystalline, consisting of micron-size grains.

\section{A. Grain Separation}

We first consider enhanced field emission due to separation of grains from a body by electric field tension acting normal to the surface. If the electric force, $F_{E}$, is high enough to overcome the binding energy, the brittle destruction tension, $\square_{\mathrm{B}}$, of the grain is separated from the body, and the sharp edge produces enhanced field emission, represented by Fig. 1. 


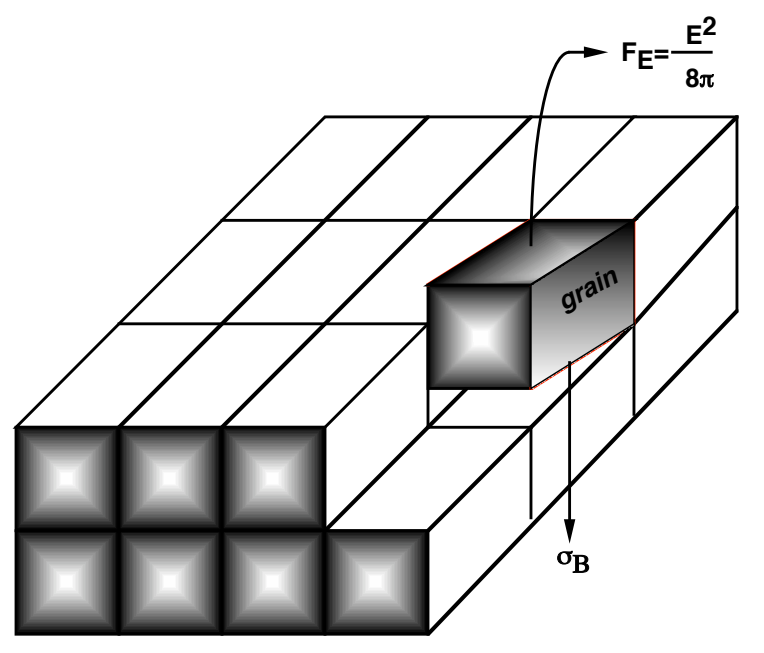

Grain separation

Fig. 1. Schematic of separation of grain from body by electric tension

Separation of one grain results in crack formation that provokes separation of the next grain, then the next and so on (Fig. 2). The enhanced electric field results from a displaced linear protrusion.

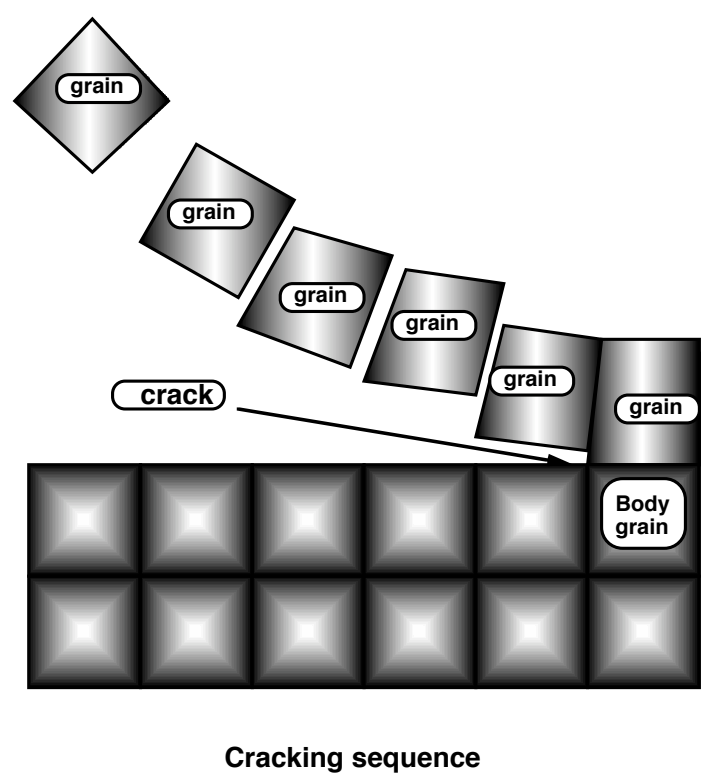

Fig. 2. Schematic of grain-by-grain separation

\section{B. Maximum Electric Field}

The above kind of brittle destruction takes place by the stripping of grains one by one. The binding force can be estimated by the threshold force, i.e., yield of strength $\square_{\mathrm{B}}$. For example, 
for a Be electrode, $\square_{\mathrm{B}}=1.4-4.5 \mathrm{katm}$, depending on the material kind. We assume the $\mathrm{Be}$ electrode has $\square_{\mathrm{B}}=4 \mathrm{katm}$. The electric field force is

$$
\vec{F}_{E}=\frac{1}{2} \square \vec{E}=\frac{E^{2}}{8 \square} \vec{n}
$$

This force, $F_{E}$, should exceed the strength, $\square_{B}$, to detach a grain from the body. The corresponding electric field at which the grain can separate from the body is

$$
\frac{E_{\max }^{2}}{8 \square}=\square_{B}, E_{\max }=\sqrt{8 \square \square_{B}}, \quad E_{\max }\left(\square_{B}=4 \mathrm{katm}\right)=10 \frac{\mathrm{GeV}}{\mathrm{m}}
$$

To estimate the electric field near a grain with size $a$, one can regard the electric field of a conductive hemicylinder in the homogeneous electric field $\mathrm{E}_{0}$. The electric field of a hemicylinder displaced on the surface with potential $\mathrm{U}_{0}$ and electric field $\mathrm{E}_{0}$ can be described by the superposition of two fields: from plane surfaces (electrodes) and an infinitive cylinder. See Figs. 3 and 4.

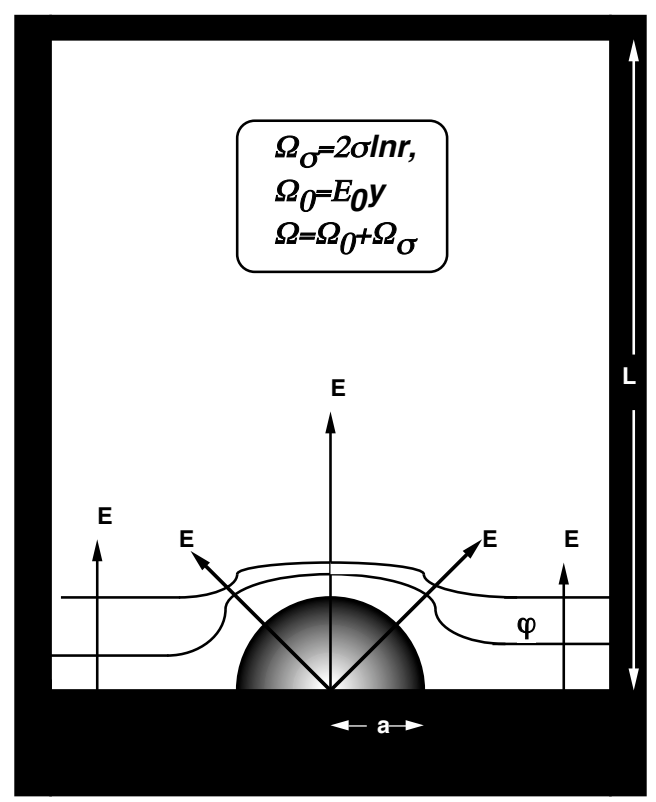

Fig. 3. Equipotential lines and electric fields for hemicylinder

The potentials corresponding to electric fields are

$$
\square=\square_{0}+\square_{\square} \text {, where } \square_{0}=E_{0} y \text {, and } \square_{\square}=2 \square \ln r
$$

The electric fields are 


$$
\begin{aligned}
& E_{x}=\frac{\partial \square}{\partial x}=\square \frac{2 x}{x^{2}+y^{2}}, E_{y}=\frac{\partial \square}{\partial y}=\square \frac{2 y}{x^{2}+y^{2}}+E_{0} \\
& E \square E_{0} \sqrt{1+\frac{1}{\ln ^{2} a}\left(\frac{L}{a}\right)^{2} \frac{a^{2}}{r^{2}}+\frac{1}{\ln a} \frac{L}{a} \frac{a y}{r^{2}}}, \square=\frac{L}{a}, \\
& E_{r=a} \square E_{0} \sqrt{1+\frac{1}{\ln ^{2} a} \square^{2}+\frac{1}{\ln a} \square \sin \square}
\end{aligned}
$$

with $\square$ being the surface charge density. For $L>>$ a, i.e., $\square>>1$,

$$
\begin{aligned}
& E_{r=a} \square E_{0} \frac{1}{\ln a} \square>>E_{0}, L=1 \mathrm{~cm}, a=10^{\square 3} \mathrm{~cm}=10 \square \mathrm{m} \\
& \square=\frac{L}{a} \geq 10^{3}, \ln a \square 10, E_{r=a} \geq 10^{3} E_{0} \\
& E_{0} \square 10 \frac{\mathrm{MeV}}{\mathrm{m}}, \quad E_{a} \square 10 \frac{\mathrm{GeV}}{\mathrm{cm}}
\end{aligned}
$$

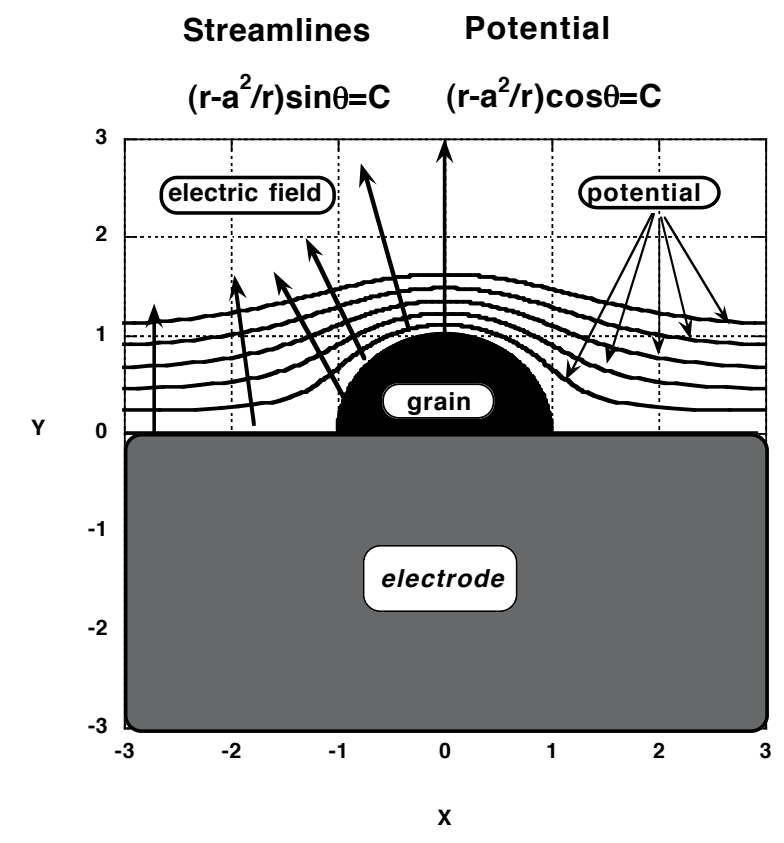

Fig. 4. Streamline and potential near single cylinder

Figure 5 shows the dependence of the field enhancement on grain size. 


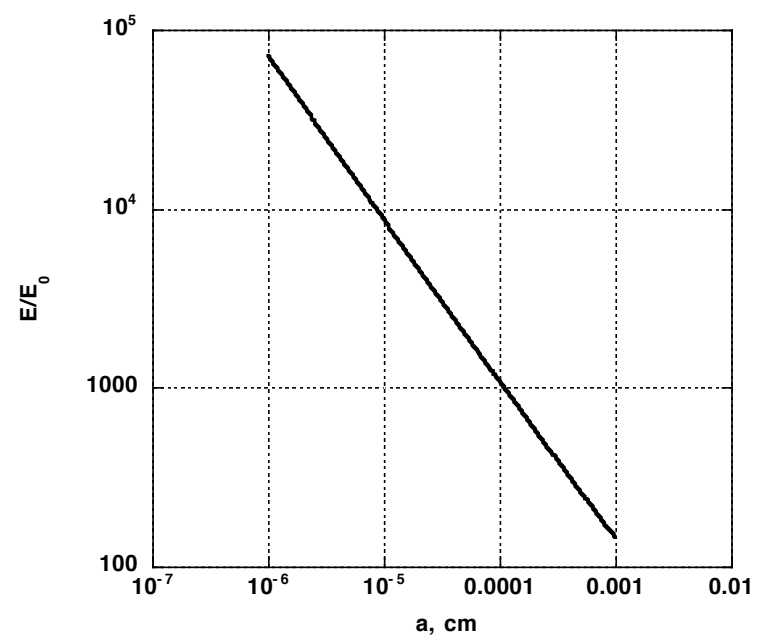

Fig 5. Field enhancement as function of grain size

The force acting on the hemicylinder is

$$
\begin{aligned}
& f_{y}=\frac{1}{a} \frac{1}{2} \square E \cos \square=\frac{1}{a} \frac{E^{2}}{8 \square} \cos \square \\
& F=\frac{1}{a} \underset{0}{\square} f_{y} a \cos \square d \square=\frac{E^{2}}{8 \square}
\end{aligned}
$$

$F(E)=\frac{E^{2}}{8 \square}=40 E^{2}, F(10)=4 \mathrm{katm}$,

with $E$ in $\frac{G e V}{m}$, and $F$ in atm.

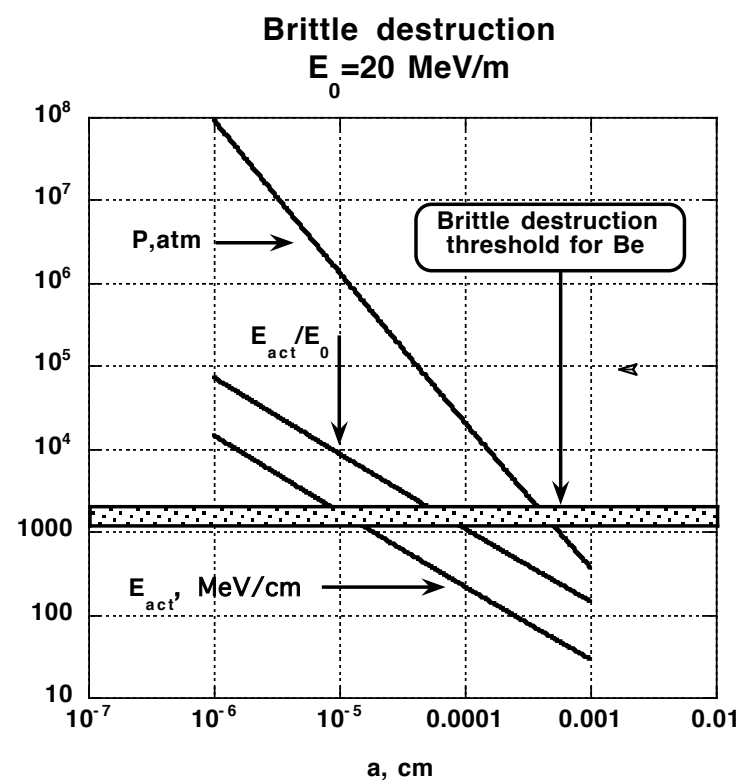

Fig. 6. Destruction limit as function of grain size 


\section{Maximum Current}

The maximum available current is determined by the number of spots (i.e., points in which grains are separated) and the maximum electric field $\left(\mathrm{E}_{\max }\right)$, which is, determined by the minimum size of the grains detached during the brittle destruction.

\section{Minimum Size of Grains}

As the electric field of grains is determined by the size of the grains, $a$, the minimum available grain size can be estimated from

$$
\begin{aligned}
& E_{\mathrm{act}}=E_{0} \frac{L}{a} \frac{1}{|\ln a|}=E_{\max }, \square=a|\ln a|=L \frac{E_{0}}{E_{\max }} \\
& a \square 10^{\square \frac{1}{5}} \square^{\frac{4}{5}}
\end{aligned}
$$

\section{E. Maximum Number of Separating Grains}

The charge conservation law determines the maximum available number of grains. The total charge of the electrode, $\mathrm{Q}_{0}$, is determined by the electric field $\mathrm{E}_{0}$ of the wave and the area $\mathrm{S}_{0}=\square \mathrm{R}^{2}$, with $\mathrm{R}$ being the electrode radius. Actually, the maximum electrostatic energy of the wave is equal to the total wave energy because, at the maximum electric field, the linac cell is a condenser bank.

Thus,

$$
Q_{0}=\square R^{2} \square_{0}=\square R^{2} \frac{E_{0}}{4 \square}
$$

At the inhomogeneous distribution of the surface charge density $\square(r, \square)$, due to existing grains (protrusions), surface charge concentrates on the grains. In the ultimate case, all charge displaces on the grain surfaces with zero surface charge density between grains that can be regarded as current source spots.

The maximum available number of such spots, $\mathrm{N}_{\text {spot }}$, and full area of spots, $\mathrm{S}_{\text {spot, }}$ can be estimated by assuming all charge is displaced on the spots (ultimate case):

$$
\begin{aligned}
& Q_{0}=2 \square a^{2} \square_{\text {spot }} N_{\text {spot }}, \square_{\text {spot }}=\frac{E_{\max }}{4 \square}, Q_{\text {spot }}=2 \square a^{2} \square_{\text {spot }} \\
& N_{\text {spot }}=\frac{Q_{0}}{Q_{\text {spot }}}=\frac{R^{2}}{4 a^{2}} \frac{E_{0}}{E_{\max }} \\
& S_{\text {spot }}=\square a^{2} N_{\text {spot }}=\square R^{2} \frac{E_{0}}{E_{\max }} \ll \square R^{2}
\end{aligned}
$$

The total number of spots, $\mathrm{N}_{\text {spot}}$, can be very high, but the density of spots, $\mathrm{n}_{\text {spot }}$, is not large (Table 1). Nevertheless, the total current, $I_{\max }$, ejected from these spots can be very high due to the exponential character of the field emission dependence on $\mathrm{E}_{\text {act }}$. 
Table 1. Parameters for current source spots

$E_{\max }=10 \frac{\mathrm{GeV}}{\mathrm{m}}, E_{0}=10 \frac{\mathrm{MeV}}{\mathrm{m}}, T M_{010}-\operatorname{mode}, R=2.405 \mathrm{~L}$

\begin{tabular}{|c|c|c|c|c|c|c|}
\hline $\boldsymbol{f}(\mathbf{G H z})$ & $\mathbf{L}(\mathbf{c m})$ & $\mathbf{R}(\mathbf{c m})$ & $\mathbf{a}(\square \mathbf{m})$ & $\mathbf{N}_{\text {spot }}$ & $\mathbf{n}_{\text {spot }}\left(\mathbf{c m}^{-2}\right)$ & $\mathbf{S}_{\text {spot }}\left(\mathbf{c m}^{2}\right)$ \\
\hline 0.5 & 30 & 72 & 60 & $0.36 \cdot 10^{5}$ & 2.2 & 4 \\
\hline 1.0 & 15 & 36 & 30 & $1.44 \cdot 10^{5}$ & 8.8 & 1 \\
\hline 5.0 & 3 & 7.2 & 2 & $0.64 \cdot 10^{5}$ & 2000 & 0.04 \\
\hline
\end{tabular}

\section{F. Fowler-Nordheim Field Emission Current}

These spots eject current due to the field emission according to $\mathrm{E}_{\mathrm{act}}=\mathrm{E}_{\max }$. The fit formula for the Fowler-Nordheim emission current density, $\mathrm{j}^{\mathrm{FN}}$, is

$$
j^{F N}=1.4 \cdot 10^{\square 6} \frac{E^{2}}{\square} \cdot 10^{\frac{4.39}{\sqrt{\square}}} \cdot 10^{\square 2.82 \cdot 10^{7}} \frac{\square^{\frac{3}{2}}}{E}
$$

where $j$ is current density $\left(\mathrm{A} / \mathrm{cm}^{2}\right), \mathrm{E}$ is electric field $(\mathrm{eV} / \mathrm{cm})$, and $\mathrm{Q}$ is the potential barrier $(\mathrm{eV})$. In Figs. 7 and 8, the current density is given for $\square=4.5 \mathrm{eV}$ as a function of acting energy, $\mathrm{E}_{\text {act, }}$ and spot size a.

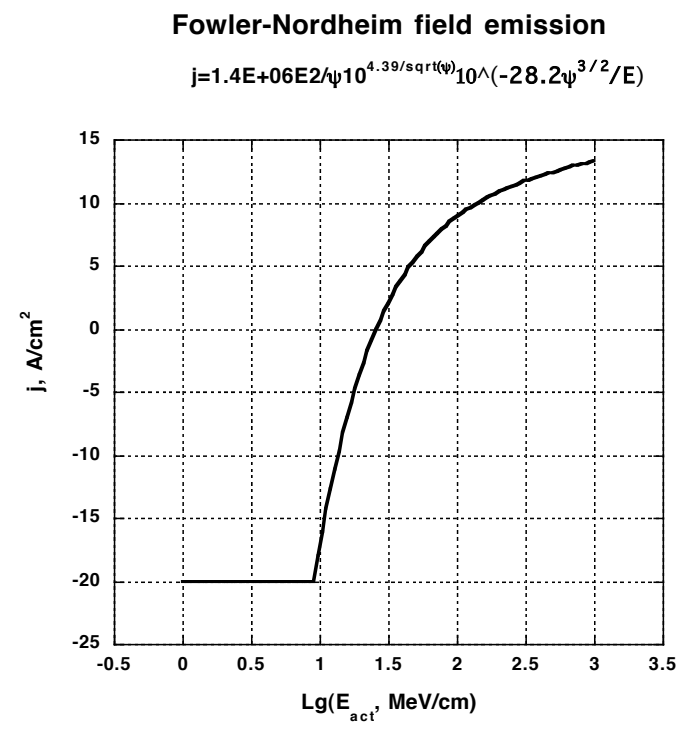

Fig. 7. Fowler-Nordheim field emission current 


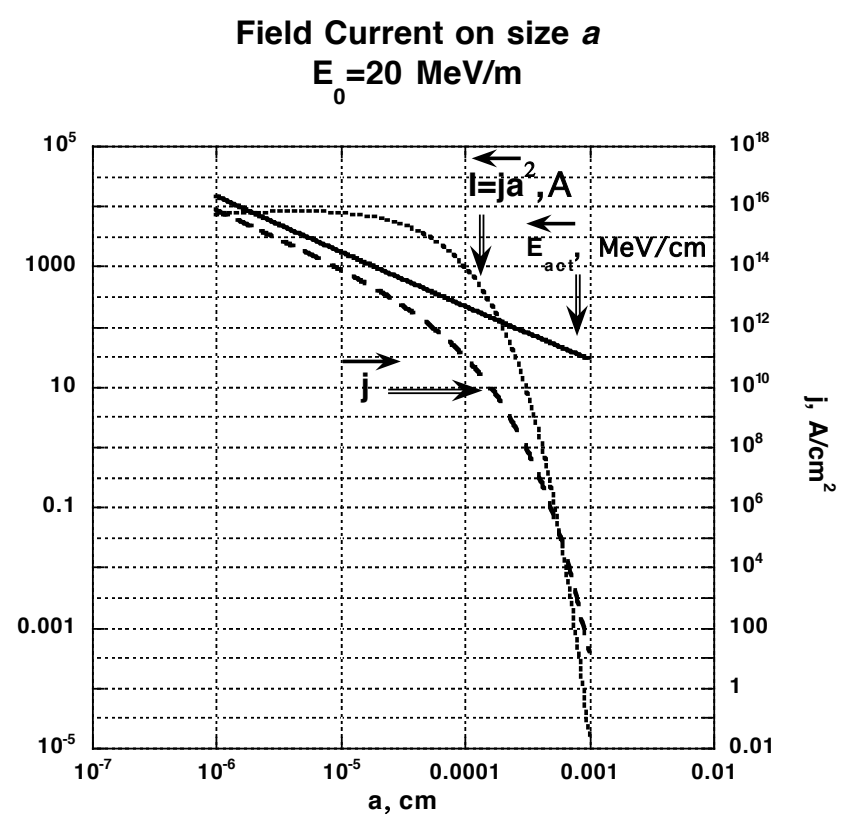

Fig. 8. Dependence of the field emission on the spot size

The corresponding current through one spot, $\mathrm{i}_{\text {spot, }}$ and the total maximum current, $\mathrm{I}_{\max }$, are

$$
I_{\max }=j_{\text {spot }}\left(E_{\max }\right) S_{\text {spot }}=j_{\text {spot }}^{F N}\left[R^{2} \frac{E_{0}}{E_{\max }}, i_{\text {spot }}^{F N}=\square a^{2} \cdot j_{\text {spot }}^{F N}\right.
$$

For $\mathrm{E}_{\max }=10 \mathrm{GeV} / \mathrm{m}$,

$$
I_{\max }=2.86 \cdot 10^{10} A, i_{\text {spot }}^{F N}=21 k A
$$

Such spot current corresponds to the following magnetic field $\left(\mathrm{B}_{\text {spot }}\right)$, magnetic pressure $\left(\mathrm{P}_{\square}\right)$, and temperature $(\mathrm{T})$ :

$$
\begin{aligned}
& B_{\text {spot }}=\frac{0.2 i_{\text {spot }}^{F N}}{a}=1.33 \cdot 10^{6} \text { gauss, } \quad P_{\square}=\frac{B_{\text {spot }}^{2}}{8 \square}=70 \mathrm{katm} \\
& T=\frac{P_{\square}}{3 n k}=1375 \mathrm{~K}
\end{aligned}
$$

Table 2 shows these values for two other spot currents (84 and $0.2 \square \mathrm{A}$ ). In addition, such magnetic force acting in the r-direction helps in the detachment of pieces.

Table 2. Magnetic pressure and corresponding temperature

$E_{\max }=10 \frac{G e V}{m}, E_{0}=10 \frac{M e V}{m}, T M_{010} \bmod e, R=2.405 \mathrm{~L}$ 


\begin{tabular}{|c|c|c|c|c|c|c|c|}
\hline $\begin{array}{c}f \\
(\mathbf{G H z})\end{array}$ & $\begin{array}{c}\mathbf{L} \\
(\mathbf{c m})\end{array}$ & $\begin{array}{c}\mathbf{R} \\
(\mathbf{c m})\end{array}$ & $\begin{array}{c}\mathbf{a} \\
(\boldsymbol{\mu m})\end{array}$ & $\begin{array}{c}\boldsymbol{i}_{\text {spot }} \mathbf{F N} \\
(\boldsymbol{k} \boldsymbol{A})\end{array}$ & $\begin{array}{c}\mathbf{B} \\
(\text { Mgauss })\end{array}$ & $\begin{array}{c}\mathbf{P}_{\boldsymbol{\mu}} \\
(\boldsymbol{k a t m})\end{array}$ & $\begin{array}{c}\mathbf{T} \\
(\boldsymbol{K})\end{array}$ \\
\hline 0.5 & 30 & 72 & 60 & 84 & 2.6 & 280 & 5500 \\
\hline 1.0 & 15 & 36 & 30 & 21 & 1.3 & 70 & 1375 \\
\hline 5.0 & 3 & 7.2 & 2 & 0.2 & 11.9 & 15750 & 310000 \\
\hline
\end{tabular}

\section{G. Child-Langmuir Emission Current}

The time for spot discharging according to the Fowler-Nordheim formula is

$$
\begin{aligned}
& q_{\text {spot }}=\square_{\text {spot }} \square a^{2}=\frac{E}{4 \square} \square a^{2}=\frac{1}{4} 10^{\square 9} \text { coulomb } \\
& \square_{\text {discharge }}=\frac{q_{\text {spot }}}{i_{\text {spot }}^{F N}}=1.2 \cdot 10^{\square 14} \mathrm{~s} \\
& \square_{\text {charge }}=c \square_{\text {discharge }}=3.6 \cdot 10^{\square 4} \mathrm{~cm}
\end{aligned}
$$

These results mean that the current is so large that the spot discharges rapidly, resulting in space charge near the spot surface at a distance close to the spot radius. To determine the real current, one must solve the Poisson equation by taking into account the space charge of the flowing electron beam. The full current from the spot (protrusion) is limited by the space charge, and such a diode characteristic closely follows "a three-halves" Child-Langmuir law [8].

For a given problem, a qualitative and quantitative solution with good accuracy can be obtained by consideration of the spherical case, where the inner central electrode is a ball (and the outer electrode is the inner side of sphere with radius close to L). The Poisson equation taking into account space charge is

$$
\begin{aligned}
& \frac{1}{r^{2}} \frac{d}{d r}\left(r^{2} \frac{d \square}{d r}\right)=\square 4 \square e n(r) \\
& j_{0}=e n_{0} V_{0} 4 \square r_{0}^{2}=e n(r) V 4 \square r^{2} \\
& \frac{m V^{2}}{2} \square e \square=\frac{m V_{0}^{2}}{2}, V^{2}=V_{0}^{2}+\frac{2 e \square}{m}, V_{0}^{2}=\frac{2 e \square \square}{m}
\end{aligned}
$$

where $e$ is the electron charge, $n$ is the density, $V$ is the velocity, and $\square$ is the potential; subscript 0 relates to the values at the surface. In dimensionless form, the Poisson equation is

$$
\begin{aligned}
& x=\frac{r}{r_{0}}, \square=\frac{\square}{\square_{0}}, \\
& \frac{d}{d x}=x^{2} \frac{d \square}{d x}=\frac{1}{\sqrt{\square \square+\square}}, \square(x=1)=0
\end{aligned}
$$

or 


$$
\begin{aligned}
& \frac{d^{2} \square}{d x}=\square \frac{1}{\sqrt{x(\square \square+\square)}}, \square=\frac{\square}{x}, \\
& \square(x=1)=0, \quad \square \square<<1
\end{aligned}
$$

Figure 9 gives the distribution of potential vs. radius for the case without space charge (formal case) and with space charge (real case). The two curves show that the space charge does not change the potential shape very much, but the current is less $(\approx 4$ times $)$.

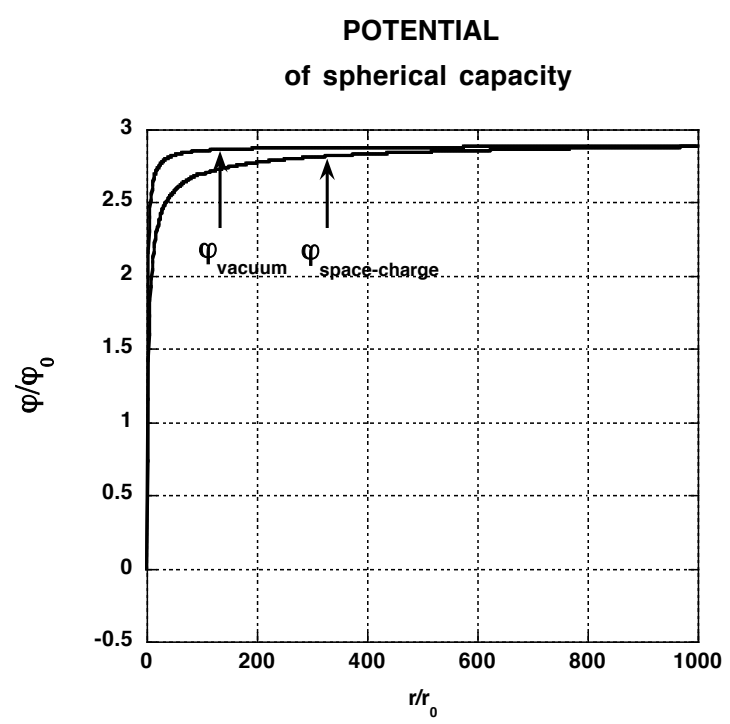

Fig. 9. Potential of spherical capacity with and without space charge

The Child-Langmuir total current and current density from one spot are

$$
i_{\text {spot }}^{C L}=A U^{3 / 2}, A=2.62 \cdot 10^{\square 6}, j_{\text {spot }}^{C L}=A \frac{U^{3 / 2}}{4 / a^{2}}
$$

where $\mathrm{U}$ is the potential between electrodes, and $A$ is constant.

Such current can heat the spot to a very high temperature. The spot temperature is determined by the equilibrium between joule heating and cooling by heat conduction with a characteristic length close to the spot size $a$ :

$$
c_{v} \frac{d T}{d t}=\square j^{2} \square \frac{\square T}{a^{2}}, T=\frac{\square j^{2} a^{2}}{\square}=\frac{\square i^{2}}{\square a^{2}}
$$

where $\square$ is the electric resistance, $\square$ is heat conduction, $i$ is current $(k A)$, and $T$ is temperature $(K)$. For Be with $a=30 \square \mathrm{m}, \square=1.35 \cdot 10^{\square 8} \square \cdot \mathrm{cm}$, and $\square=2 \frac{\mathrm{W}}{\mathrm{cm}}, T=0.75 \cdot 10^{3} i^{2}$.

As the Child-Langmuir current is less than the Fowler-Nordheim current, the above assumption of unlimited emission is valid. Table 3 shows our calculations for various conditions using the Child-Langmuir equations. 
Table 3. Spot parameters according to the Child-Langmuir current

$E_{\max }=10 \frac{\mathrm{GeV}}{\mathrm{m}}, E_{0}=10 \frac{\mathrm{MeV}}{\mathrm{m}}, T M_{010}$ mode, $R=2.405 \mathrm{~L}$
\begin{tabular}{|c|c|c|c|c|c|c|}
\hline $\boldsymbol{f}(\boldsymbol{G H} \boldsymbol{z})$ & $\mathbf{L}(\boldsymbol{c m})$ & $\mathbf{R}(\boldsymbol{c m})$ & $\mathbf{A}(\boldsymbol{\mu m})$ & $\boldsymbol{i}_{\text {spot }} \mathbf{F n}(\boldsymbol{k A})$ & $\boldsymbol{i}_{\text {spot }} \mathbf{C L}(\boldsymbol{k A})$ & $\mathbf{T}(\boldsymbol{e V})$ \\
\hline 0.5 & 30 & 72 & 60 & 84 & 9.6 & 1.75 \\
\hline 1.0 & 15 & 36 & 30 & 21 & 4.8 & 0.43 \\
\hline 5.0 & 3 & 7.2 & 2 & 0.2 & 0.046 & 0.002 \\
\hline
\end{tabular}

At a temperature of a few eV, a vapor cloud forms near the spot surface with probably some ionized cloud ahead of the vapor cloud. We conclude that at $\mathrm{f}=1 \mathrm{GHz}, \mathrm{E}>10 \mathrm{MeV} / \mathrm{m}$, and $\mathrm{U}=1.5$ $\mathrm{MeV}$, breakdown can occur due to heating of grains (spots) in a process like "micro-explosion" breakdown [2].

\section{BREAKDOWN SUMMARY}

One reason for the breakdown is separation of grains from the body by electric tension. The maximum electric field at the separating grain, $\mathrm{E}_{\max }$, is determined by the yield of tension, $\square_{\mathrm{B}}$. The current ejected by a separate grain is determined by the "three-halves" Child-Langmuir law and is proportional to $\mathrm{U}^{3 / 2}$, where $\mathrm{U}=\mathrm{E}_{0} \mathrm{~L}, \mathrm{~L}=\square / 2$, and $\square=\mathrm{c} /$ f. The size of grains should be close to the minimum available size a, determined by $\mathrm{E}_{0}$ and $\mathrm{E}_{\max }$. The total current passing through the grain can be large enough to vaporize (and partially ionize) the grain, i.e., to heat it above the so-called ionization temperature $\mathrm{T}_{\text {ion }}$ at about $1 \mathrm{eV}$. Combining Eqs. 2.5, 2.7, and 2.17 one can obtain the breakdown condition as a function of frequency $\mathrm{f}$ and material properties such as electric resistance, $\square$; heat conductivity, $\square$; and yield of tension, $\square$ в:

$$
E_{0}>(10 \square 25) f_{G H z} \square_{B}^{\square \frac{5}{14}}=\frac{\square T_{i o n}}{\square} \overbrace{}^{\frac{4}{7}}, \frac{M e V}{m}
$$

where $\square, \square$, and $\square_{\mathrm{B}}$ are the corresponding values for Be. Figure 10 plots the upper and lower breakdown limit as a function of frequency. The breakdown electric field according to the Kilpatrick criterion is within these limits (Eq. 1.2). 


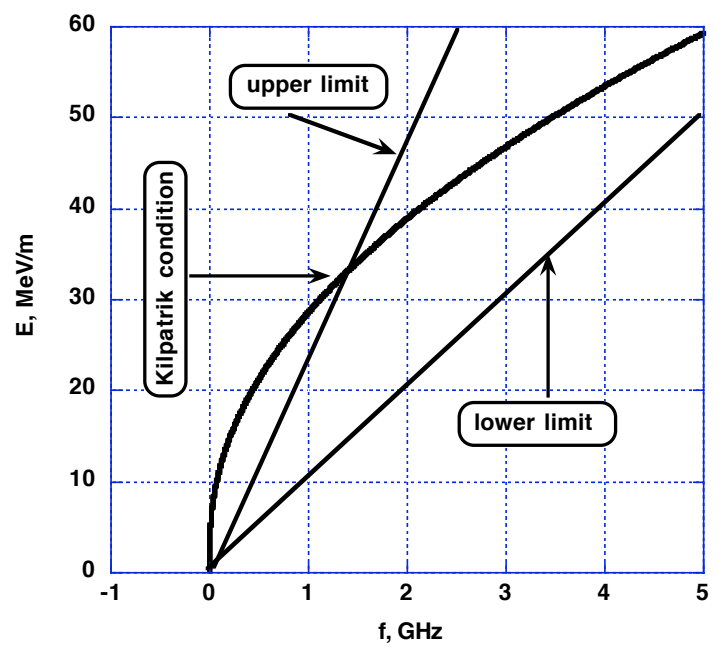

Fig. 10. Breakdown threshold as function of frequency

\section{DARK CURRENT}

The enhanced dark current, $I_{\text {dark}}$, is another concern of the linacs. Figure 11 shows results from measurements of radiation fluxes coming out through the electrode [9]. The intensity, I, of radiation is linearly proportional to the dark current.

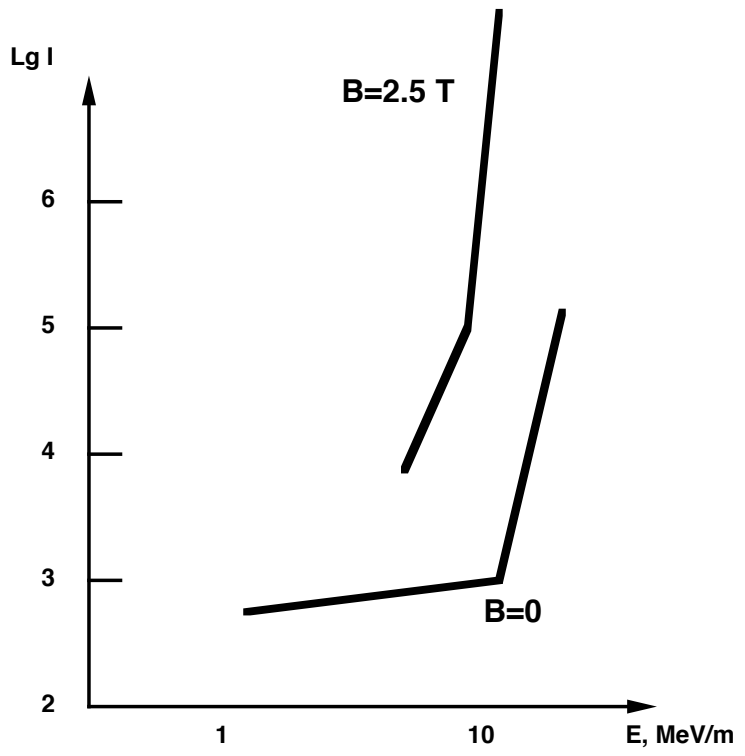

Fig. 11. Effect of radiation fluxes through electrode on electric field magnitude

Three observations about dark current were made:

1. dark current exceeds the Fowler-Nordheim current by many orders of magnitude,

2. dark current increases so fast with the applied electric field $\left(I_{\text {dark }} \mu E_{0}{ }^{n}, n \approx 10>>1\right)$ that the increase of dark current can be regarded as a process with a threshold, and

3. including a guide magnetic field increases the dark current by many orders of magnitude and shifts the threshold to a lower value. 
Physical processes not considered before need to be taken into account in the results. Below, some qualitative considerations for understanding such processes are given. One of the main processes, from our point of view, is the transition of the ejected electrons accelerated in the linacs to relativistic and ultrarelativistic energies. Such a transition does not involve simple energy increases, because relativistic electron behavior differs qualitatively from nonrelativistic due to a) change in the character of the stopping power, b) the velocity limitation of the speed of light, and c) the strong influence of the guide magnetic field.

\section{A. Stopping Power of the Relativistic Electrons and Secondary Emissions}

The dark current arises due to the following three processes: 1) field emission--acceleration of ejected electrons (named "primary" in the following text) in the linac electric field, to energy close to the applied voltage $\left.\left(\mathrm{U}_{0}=\mathrm{E}_{0} \mathrm{~L}\right) ; 2\right)$ secondary electrons--coming into being due to interaction of primary electrons with the opposite electrode or walls; and 3) the so-called "multipactor" effect--when the electrons are also accelerated in synchronization with the rf linac electric field. The main concern is the coefficient of secondary emission $\square$, where $\mathrm{n}_{\text {secondary }}$ includes both reflected primary electrons and electrons coming into being due to ionization, i.e., the "true" secondary electrons. The number of reflected primary electrons is much less than that of the true secondary electrons; usually $\mathrm{n}_{\text {secondary }}$ is close to the density of the true secondary electrons. A complete theory of secondary electron emission is absent up to now, but numerous experimental data more or less coincide with some partial theories. Most of these data are for relatively low energies (keV range), i.e., nonrelativistic electron beams.

It is well known that $\square$ in insulators is very large. The value of $\square$ can be $\approx 100$ and more, but in metals $\square$ is $<1$ due to the large range of electrons, $\square_{\mathrm{e}}$. Thus, secondary electrons are borne at rather long distances from the target surface. Most of these secondary electrons have low energy, $<50 \mathrm{eV}$, and cannot leave the metal because of strong energy losses due to interaction with electrons in the conducting zone. See Fig. 12.

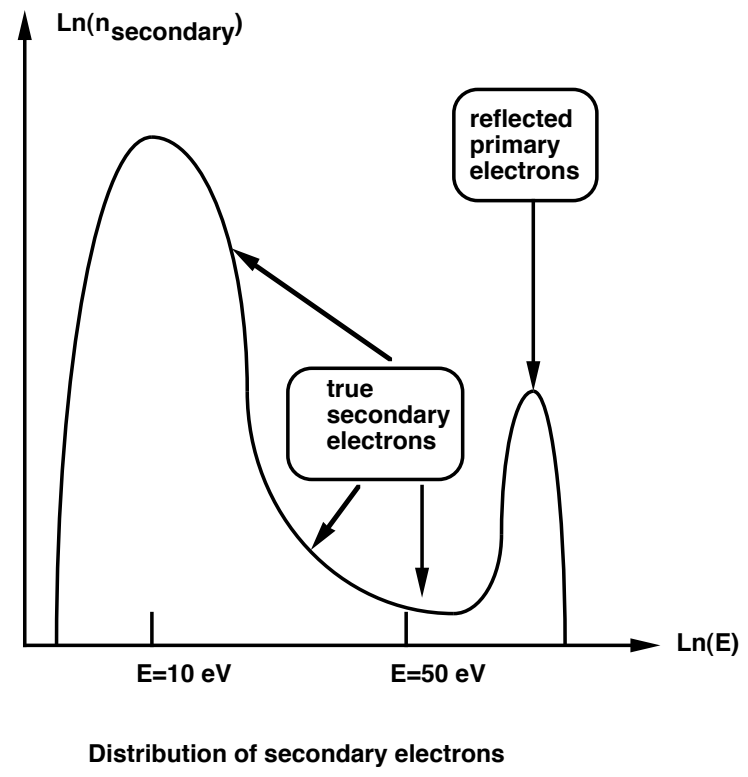

Fig. 12. Energetic spectrum of the secondary electrons 
The path lengths of the nonrelativistic and ultrarelativistic electrons are given by

$$
\begin{aligned}
& \square_{e, \text { nonrel }}=\frac{E}{\frac{d E}{d x}}=\frac{2}{4 \square n e^{e} e^{4}} \frac{1}{\ln \frac{E}{\bar{I}}} E^{2},
\end{aligned}
$$

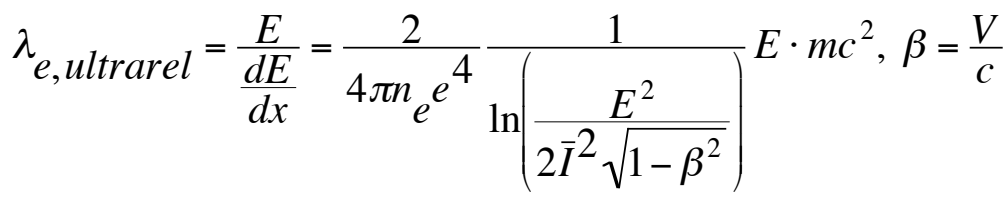

where $\bar{I} \square$ mean ionization potential

Thus, the path length of the ultrarelativistic electron is relatively low:

$$
\frac{\square_{e, \text { nonrel }}}{\square_{e, \text { ultrarel }}} \square \frac{E}{m c^{2}}>>1
$$

The secondary emission coefficient $\square$ for the ion is more than that for the electron with the same energy. It is well known in fission science that $\square$ of fragments exceeds one, and reaches 10-100 because of the low range of electrons and, correspondingly, production of secondary electrons closer to the target surface [10].

As the beam particle loses energy due to interactions mostly with electrons, the energy losses (or stopping power $\mathrm{F}_{\text {stop}}$ ) of charged particles do not depend on particle mass, only on charge, $\mathrm{Z}$, and velocity, V:

$$
\mathrm{F}_{\text {stop }}=\frac{d E}{d x}=\frac{4 \square n_{e}^{*} Z^{2} e^{4}}{\ln \frac{E}{\bar{I}}} \frac{1}{m V^{2}}, n_{e}^{*}=Z^{*} n^{*}
$$

the symbol * relates to target material. Nevertheless, the range of electrons, $\square_{\mathrm{e}}$, is much more than the range of ions, $\square_{i}$, with the same kinetic energy, E,

$$
\frac{\square_{e}}{\square_{i}}=\frac{Z^{2} M}{m}=1835 \cdot A Z^{2} \square 2000
$$

with A being the atomic weight. Short-range ions produce secondary electrons at short distance from the target surface, and they may have enough energy to reach the target surface despite their intensive energy losses from electrons in the conductive zone. This is why ions have larger $\square$ than electrons in the nonrelativistic case: $\mathrm{E}_{\mathrm{e}}<\mathrm{mc}^{2}, \mathrm{E}_{\mathrm{i}}<\mathrm{Mc}^{2}\left(\mathrm{mc}^{2}=0.51 \mathrm{MeV}, \mathrm{Mc}^{2} \geq 1 \mathrm{GeV}\right)$.

The situation changes drastically, for the case with $\mathrm{Mc}^{2}>\mathrm{E}>\mathrm{mc}^{2}$, when the ions are nonrelativistic, but the electrons are relativistic and even ultrarelativistic. The ion range is proportional to the inverse ratio of ion and electron mass, and tracks of ions are usually very short and thick. This situation, is stated, mathematically as 


$$
\begin{aligned}
& \square_{i}=\square E_{i} / \frac{d E_{i}}{d x}=\frac{E}{\frac{4 \llbracket n^{*} Z^{2} e^{4}}{m V^{2}} \ln \left(\frac{m V^{2}}{\bar{I}}\right)}=\frac{E}{\frac{2 \sqrt{ }{ }^{*} Z^{2} e^{4}}{E} \frac{M}{m} \ln \left(\frac{2 E}{\bar{I}} \frac{m}{M}\right)} \\
& \square_{i}=\square_{0} \frac{1}{Z^{2}} \frac{m}{M} \frac{\ln \left(\frac{2 E}{\bar{I}}\right)}{\ln \left(\frac{2 E}{\bar{I}} \frac{m}{M}\right)}, \quad \square_{0}=\frac{1}{2 \square n^{*} e^{4}} \frac{E^{2}}{\ln \left(\frac{2 E}{\bar{I}}\right)}
\end{aligned}
$$

The electron stopping power $\mathrm{F}_{\text {stop }}$ changes substantially for the relativistic electrons. At first, $\mathrm{F}_{\text {stop }}$ becomes higher because the electron velocity is limited by the speed of light, $\mathrm{V} \leq \mathrm{c}$. Subsequently, the relativistic compression of the electron electric field decreases in the longitudinal direction ( $\mathbb{W}$ and increases in the transverse direction $(\square)$ :

$$
E_{\mathrm{II}}=E_{\mathrm{II}, 0}\left(1 \square \square^{2}\right), E_{\square}=\frac{E_{\square 0}}{\sqrt{1 \square \square^{2}}}, \square=\frac{V}{c}
$$

Figure 13 shows the electric field for the stopped and moving charge. The compression results in less efficiency of the interaction with the field electrons, but the joint effects are positive, i.e., the resulting $\mathrm{F}_{\text {stop }}$ becomes larger.
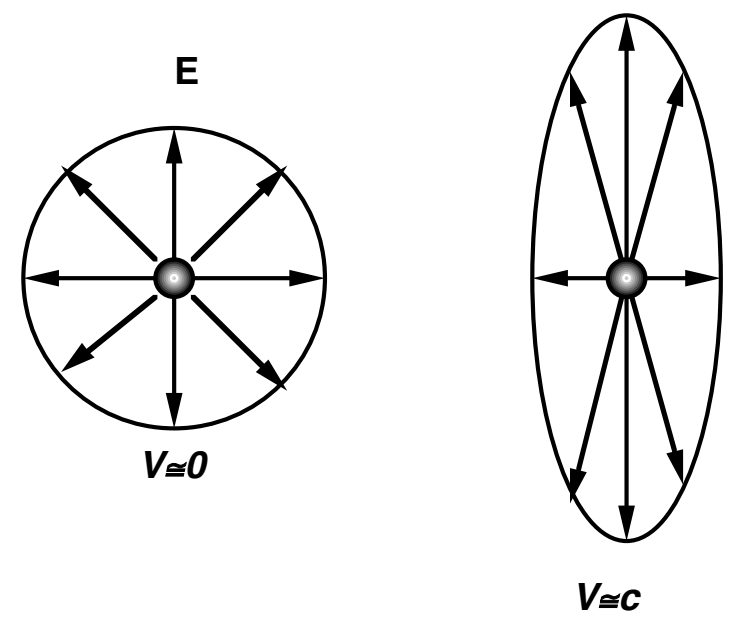

Fig. 13. Electric field of stopped and moving charge

For ultrarelativistic electrons $\mathrm{F}_{\text {stop }}$ is

$$
\square \frac{d E_{e}}{d x}=\frac{2 \square n_{0} Z e^{4}}{m c^{2}} \ln \frac{\square}{\square^{2} I^{2}} \frac{1}{\sqrt{1 \square V^{2} / c^{2}}}+\frac{1}{8}\left[\frac{\square}{\square}\right.
$$

The range with $\bar{I}$, the average ionization potential, is 


$$
\square_{e}=\square E_{e} / \frac{d E_{e}}{d x}=\square_{0} \frac{m c^{2}}{E} \frac{\ln \left(\frac{2 E}{\bar{I}}\right)}{\ln \left(\square \frac{E^{2}}{2 \bar{I}^{2}}+\frac{1}{8}\right)}
$$

Therefore, the ratio of ion and electron ranges is

$$
\frac{\square_{e}}{\square_{i}}=\frac{M c^{2}}{E} Z^{2} \frac{\ln \left(\frac{2 E}{\bar{I}} \frac{m}{M}\right)}{\ln \left(\square \frac{E^{2}}{2 \bar{I}^{2}}+\frac{1}{8}\right)}<Z^{2} \frac{M}{m} \square 2000
$$

For example, assuming

$$
E=5 \mathrm{MeV}, M=m_{p}, Z=1, \bar{I}=10 \mathrm{eV}
$$

we find that

$$
\begin{aligned}
& A=\ln \left(\frac{2 E}{\bar{I}} \frac{m}{M}\right) \square \ln 500=6.2, B=\ln \left(\square \frac{E^{2}}{2 \bar{I}^{2}}+\frac{1}{8}\right) \square \ln 2.5 \cdot 10^{12}=28.5 \\
& \frac{\square_{e}}{\square_{i}}=\frac{M c^{2}}{E} \frac{A}{B},=187.6 \frac{6.2}{28.5} \square 40
\end{aligned}
$$

with $\mathrm{A}$ and $\mathrm{B}$ variables depending on energy ionization potential and mass ratio.

Thus, the electron range is only 40 times less than the ion range. Put another way, $\square_{e}$ is only 40 times less than $\square_{i}$; thus, $\square_{\mathrm{e}}>1$ at $\square_{i}>40$.

Logically, it makes sense to take into account the photoelectric effects, because radiation losses are not small. The ratio of radiation and ionization losses is

$$
\left.\frac{d E}{d x}\right|_{\text {radiation }}:\left.\frac{d E}{d x}\right|_{\text {ionization }}=\square \square \frac{Z E}{800}
$$

with $E$ in $M e V$.

The critical energy for Be with $Z=4$ and $\square=1$ is $E_{\text {crit }} \square 200 \mathrm{MeV}$. Nevertheless, only about $1 \%$ of the energy is radiated. Photons radiated back toward the opposite electrode produce many secondary electrons due to the short range of photons.

The breakdown occurs at $\mathrm{E}=10 \mathrm{MeV} / \mathrm{m}$ for frequency $\mathrm{f}=1 \mathrm{GHz}$ with resonator size of $15 \mathrm{~cm}$. As a result, an electron coming out for any reason accelerates to $3 \mathrm{MeV}$ and becomes relativistic, which increases the secondary-electron emission coefficient above $1, \square>1$.

It would be helpful to determine the electron emission coefficients for high electron energy (more than $1 \mathrm{MeV}$ ). If the secondary emission coefficient exceeds 1 , the multipactor effect can 
take place as the resonance condition can be satisfied because electrons reach the speed of light very fast.

\section{B. Multipactor Effect with $B_{z}=0$}

To understand the influence of the multipactor effect, let us regard the simple case of a cylindrical linac with standing wave of an electric type $\left(\mathrm{TM}_{010}\right.$ mode) and with electric and magnetic fields of magnitude $\mathrm{E}_{0}\left(\mathrm{~B}_{0}=0\right)$. In this case,

$$
\begin{aligned}
& E_{z}=E_{0} I_{0}(\square r) \sin (\square t), E_{r}=0, E_{\square}=0 \\
& B_{\square}=\square E_{0} I_{1}(\square r) \cos (\square t), B_{r}=0, B_{z}=B_{0}
\end{aligned}
$$

with $\mathrm{I}_{0}$ and $\mathrm{I}_{1}$ being the Bessel functions. For a wave with frequency $\square$ and wavelength $\square$ the resonator should have length $\mathrm{L}$ and radius $\mathrm{R}$,

$$
L=\frac{\square}{2}, R=\square_{0} L, \square_{0} \square 2.405
$$

From the equation of motion, the momentum, $\mathrm{P}$, change is defined as

$$
\frac{d \vec{P}}{d t}=\frac{e}{m}\left(\vec{E}+\frac{1}{c}[\vec{V} \overrightarrow{\mathrm{B}}]\right)
$$

It follows that an electron ejected at $\mathrm{t}=0$ and radius $\mathrm{r}=0$ moves along axis $\mathrm{r}=0$ and is accelerated to a momentum, $\mathrm{P}$, and corresponding energy, $\mathrm{E}$,

$$
\begin{aligned}
& \frac{P}{m c}=\frac{2}{\square} \frac{U_{0}}{m c^{2}}, U_{0}=E_{0} L \\
& \frac{\square}{m c^{2}}=\sqrt{\left(\frac{P}{m c}\right)^{2}+1}, \frac{E}{m c^{2}}=\square \square 1, E_{\max }(\square>>1)=\frac{2}{\square} U_{0}
\end{aligned}
$$

In Fig. 14a-d, the dynamics of an electron ejected from the left electrode at different radial positions are given for frequency $\mathrm{f}=0.5 \mathrm{GHz}$ and potential between electrodes of $\mathrm{U}=10.22 \mathrm{MeV}$. Except for $\mathrm{E}$ in the right $\mathrm{y}$-axis, coordinates are given in relative values (sizes of linac); time $\square$ is the half-period, and $Z_{\max }$ is the distance at which the electron turned back or reached wall. There are three kinds of electron tracks depending on initial radial position, $r_{0}$, at the left boundary. At

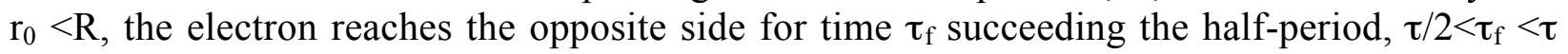
(Fig. 14a). At $\mathrm{R}_{1}<\mathrm{r}_{0}<\mathrm{R}_{2}$ the electron turns back at $\mathrm{Z}<1$ and comes back to the initial side (Fig. $14 b$ ). At $r_{0}>R_{2}$ the electron goes to the side boundary (wall) (Fig. 14c). Such significant motion in the radial direction is due to force $V_{z}, B_{\square}$ where $B_{\square}$ is the magnetic field of the wave. 


$$
f=0.5 \mathrm{GHz}, U=10.22 \mathrm{MeV}
$$

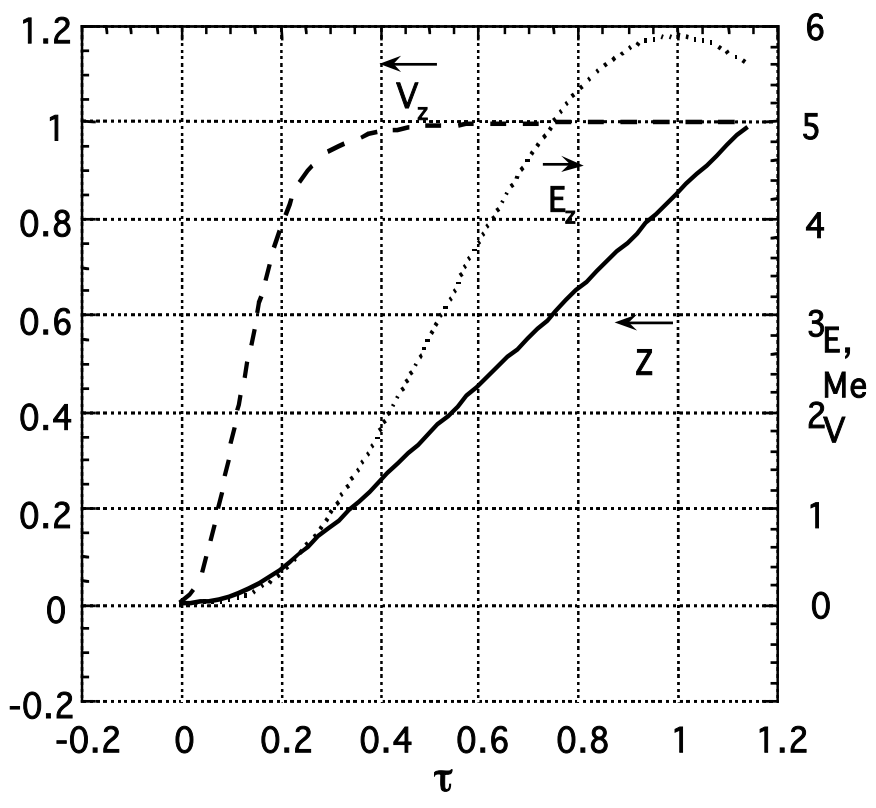

Fig. 14a. Electron ejected at $\mathrm{r}=0$ and reached the opposite electrode

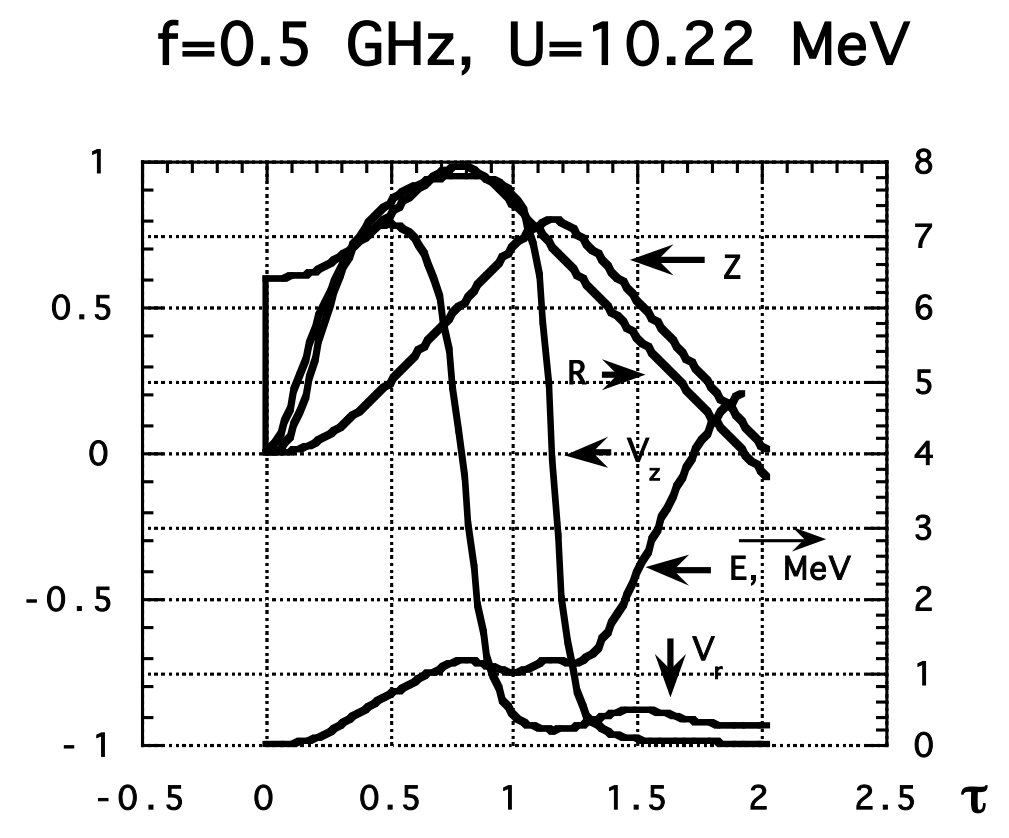

Fig. $14 \mathrm{~b}$. Electron ejected at $\mathrm{r}=0.6$ and returned at $Z_{\max }=0.8$ 


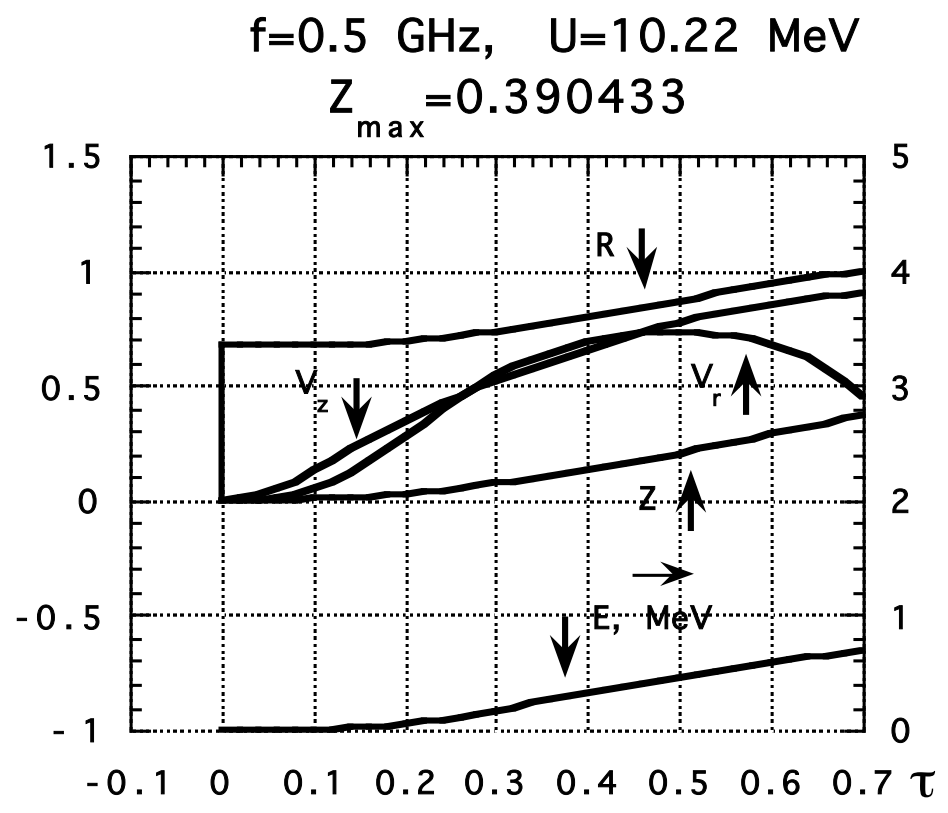

Fig. 14c. Electron ejected at $\mathrm{r}=0.675$ and reached wall at $\mathrm{Z}_{\max }=0.39$

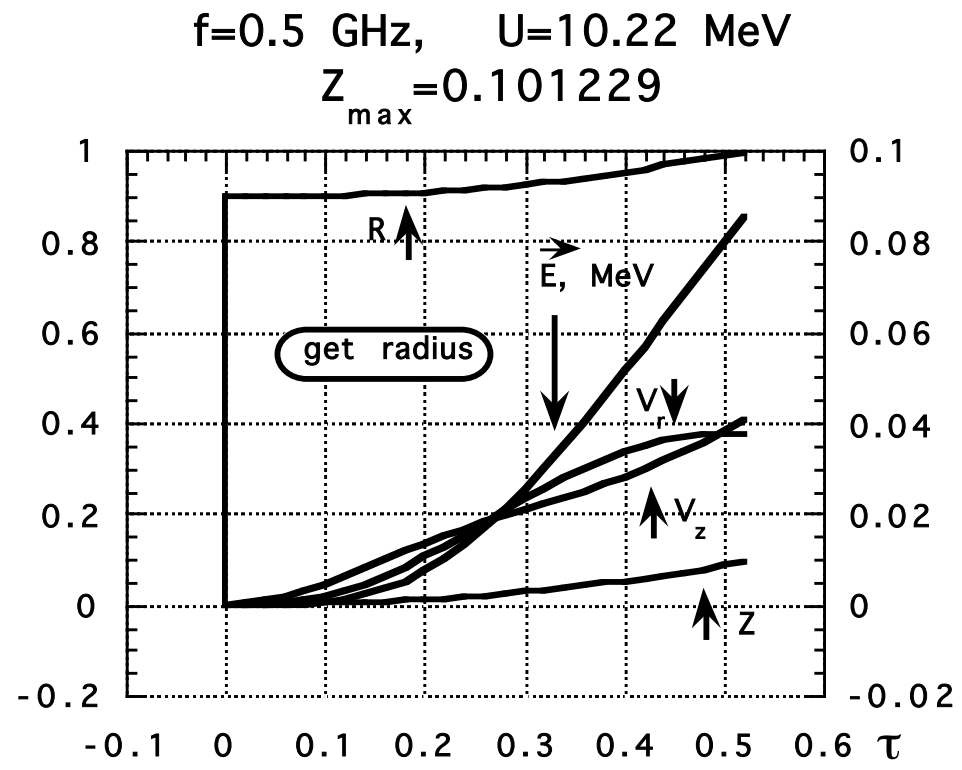

Fig. 14d. Electron ejected at $\mathrm{r}=0.9$ and reached wall at $\mathrm{Z}_{\max }=0.1$

Figure 15 is a schematic of regions illustrating different behaviors of electrons. The corresponding trajectories in the z-r plane are given in Fig. 16. 


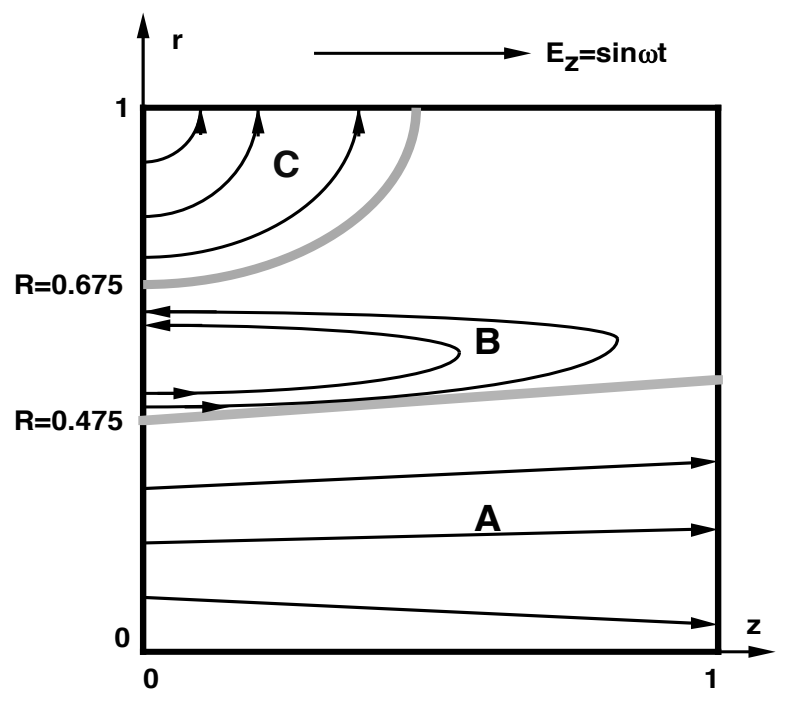

Regions of motion of electron ejected at $z=0$
A- reach opposite wall
B- back to ejecting wall
C- reach radial wall

Fig. 15. Regions of electron motion

\section{Trajectory in z-r plane} $\mathrm{U}=3 \mathrm{MeV}, \mathrm{f}=1 \mathrm{GHz}, \quad \mathrm{Z}=0$

z

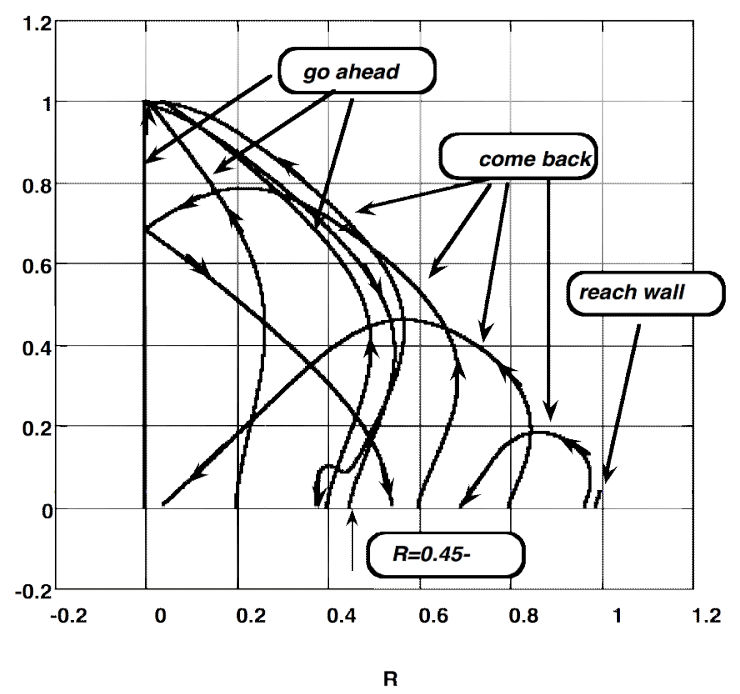

Fig. 16. Motion of electrons in z-r plane

The ejected electrons can be divided into three groups: those reaching the opposite electrode (A), returning to the left electrode (B), and going to the radial wall (C). Electrons reaching the opposite electrode have close to the maximal available energy $\left(\mathrm{E}^{\mathrm{MAx}}=6.4 \mathrm{MeV}\right)$. These electrons can produce secondary electrons. If $\square \geq 1$, new secondary electrons can accelerate at the next halfperiod and also reach the left electrode. This process can repeat many times, increasing the 
current and resulting in "dark current". Schematically, the chain process is depicted in Fig. 17. This process ends at $\mathrm{t}=22 \square$ after $\mathrm{N}_{\text {strike }}=10$ strikes when the synchronization is lost. At another frequency and electric field, $\mathrm{N}_{\text {strike }}$ can be another value. As these electrons start to move at a different phase of the wave electric field, energy changes from a few $\mathrm{keV}$ to a few $\mathrm{MeV}$. This process can end if the incoming electrons do not have enough energy to produce secondary electrons. See Fig. 18.

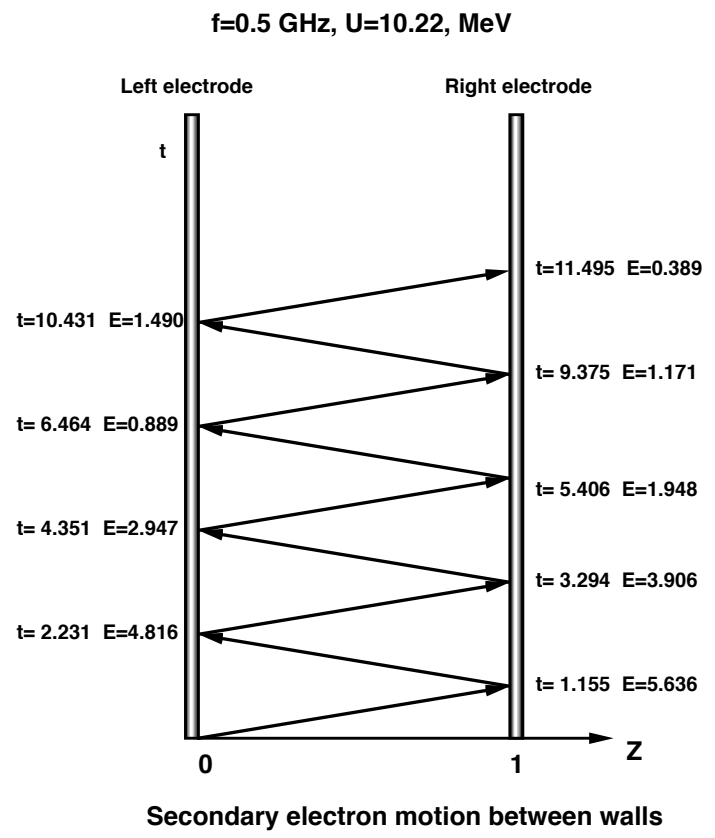

Fig. 17. Electron motion between walls at $\square=1$

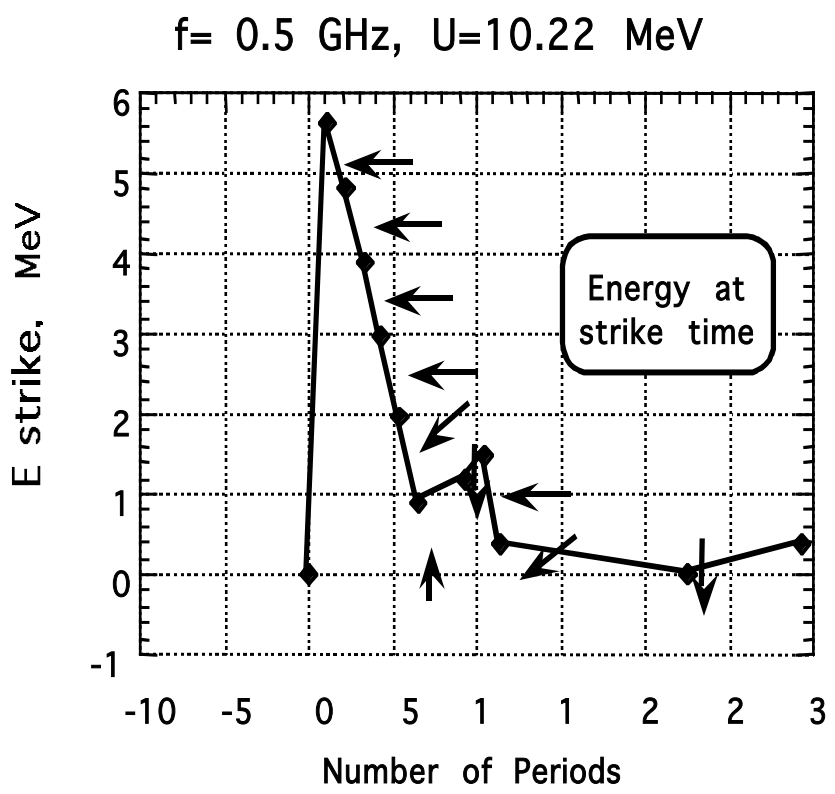

Fig. 18. Chain process of secondary electron. Points mean energy when electron strikes opposite electrode. 
It would be helpful to construct a full model of such cloud behavior between electrodes by using more or less realistic secondary-electron emission coefficients for different frequencies and electric fields. Such a model can be used to understand the dramatic increase of dark current in the linac without the guide magnetic field.

\section{Multipactor Effect with $\mathbf{B}_{\boldsymbol{z}} \neq \mathbf{0}$}

To understand why the guide magnetic field results in a drastic decrease of the dark current threshold, we developed a model for electron dynamics in the linac with strong magnetic field. Figures 19 and 20 show the electron dynamics calculated without and with the guide magnetic field, respectively.

\section{Electron dynamics}

$\mathrm{U}=3 \mathrm{Mev}, \mathrm{f}=1 \mathrm{GHz}, \mathrm{B}=0, \mathrm{R}=0.5$

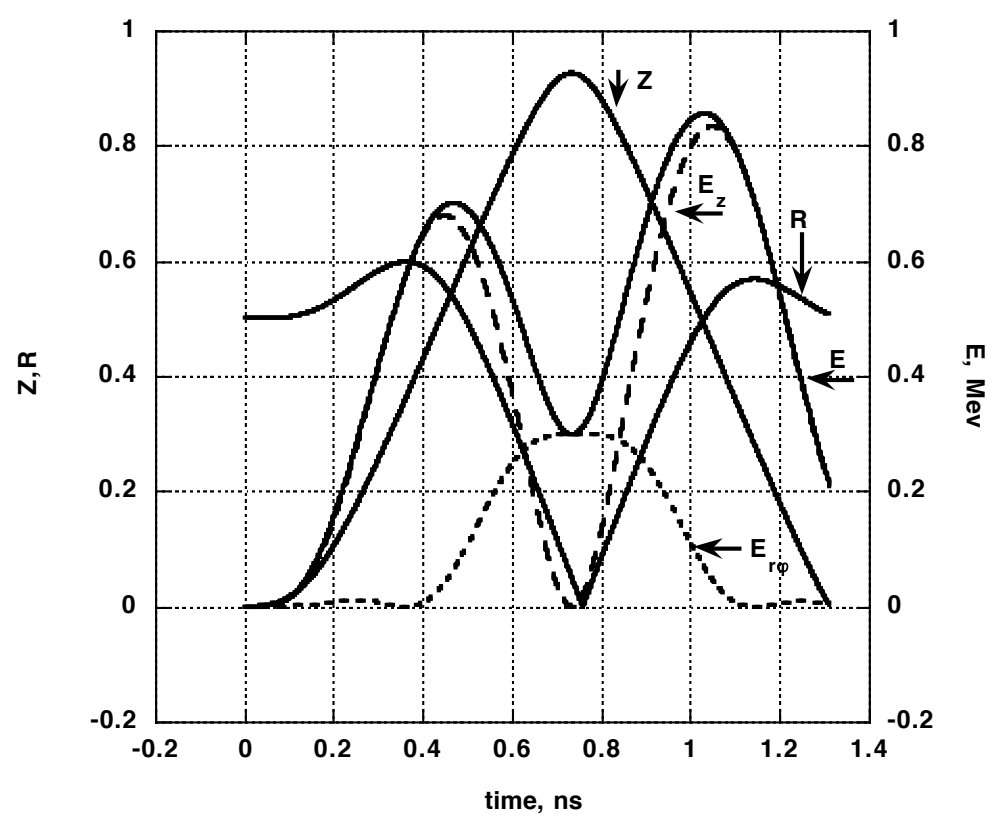

Fig. 19. Electron dynamics without guide magnetic field

The Larmor frequency for the guide magnetic field, $\square_{\mathrm{B}}\left(\mathrm{B}_{\mathrm{z}}=2.5 \mathrm{~T}\right)=440 \mathrm{GHz}$, is much more than that for the wave magnetic field, $\square_{\mathrm{B}}\left(\mathrm{B}_{\mathrm{wave}}=0.067 \mathrm{~T}\right)=11.76 \mathrm{GHz}$, and the wave frequency is $\square_{0}<<\square_{\mathrm{B}}\left(\mathrm{B}_{\text {wave }}<<\square_{\mathrm{B}}\left(\mathrm{B}_{\mathrm{Z}}\right)\right.$. As expected, the guide magnetic field results in most electrons at $\mathrm{R}<0.99$ reaching the opposite electrode. Compare Figs. 19 and 20. 


\section{Electron dynamics}

$\mathrm{U}=3 \mathrm{MeV}, \mathrm{f}=1 \mathrm{GHz}, \mathrm{B}=2.5 \mathrm{~T}, \mathrm{R}=0.5$

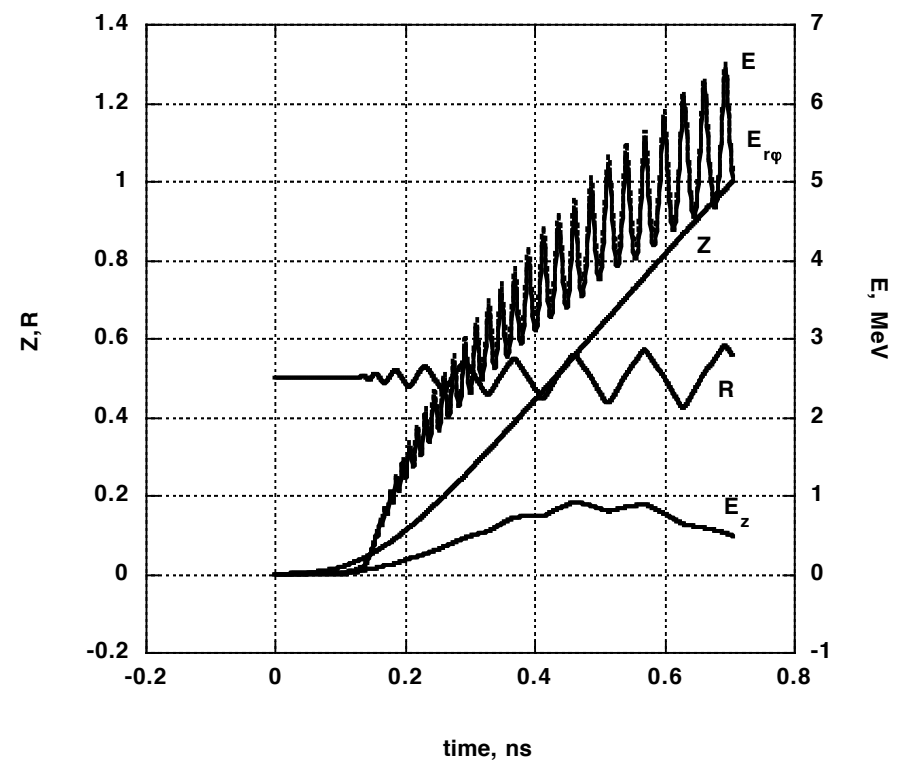

Fig. 20. Electron dynamics with guide magnetic field $\left(\mathrm{B}_{\mathrm{z}}=2.5 \mathrm{~T}\right)$

The main difference is that the total energy the electron received from the electric field increases because the gained momentum in the $\mathrm{z}$-direction, $\mathrm{P}_{\mathrm{z}}$, immediately transforms into momentum in the $\square$ and $r$ directions due to the corresponding forces:

$$
F_{r}=\frac{e}{c} \nabla_{\square}{ }^{B} \square V_{z} B_{\square} F_{\square}=\square \frac{e}{c} V_{r} B_{z}
$$

Thus, the electron trajectory in the plane is a spiral with increasing radius, i.e., momentum $\mathrm{P}_{\square}$. Electrons magnetized into a magnetic field with Larmor radius $\mathrm{Z}_{H i}<\mathrm{R}$ move strongly along the guide magnetic field; thus, all ejected electrons reach the opposite electrode. This behavior increases the number of primary electrons that produce the secondary ones because almost all electrons reach the opposite side (Fig. 21). 
Electron dynamics

$\mathrm{U}=3 \mathrm{MeV}, \mathrm{f}=1 \mathrm{GHz}, \mathrm{B}=2.5 \mathrm{~T}, \mathrm{R}_{0}=0.85$

$R, Z$ in relative units, $E$ in $M e V$

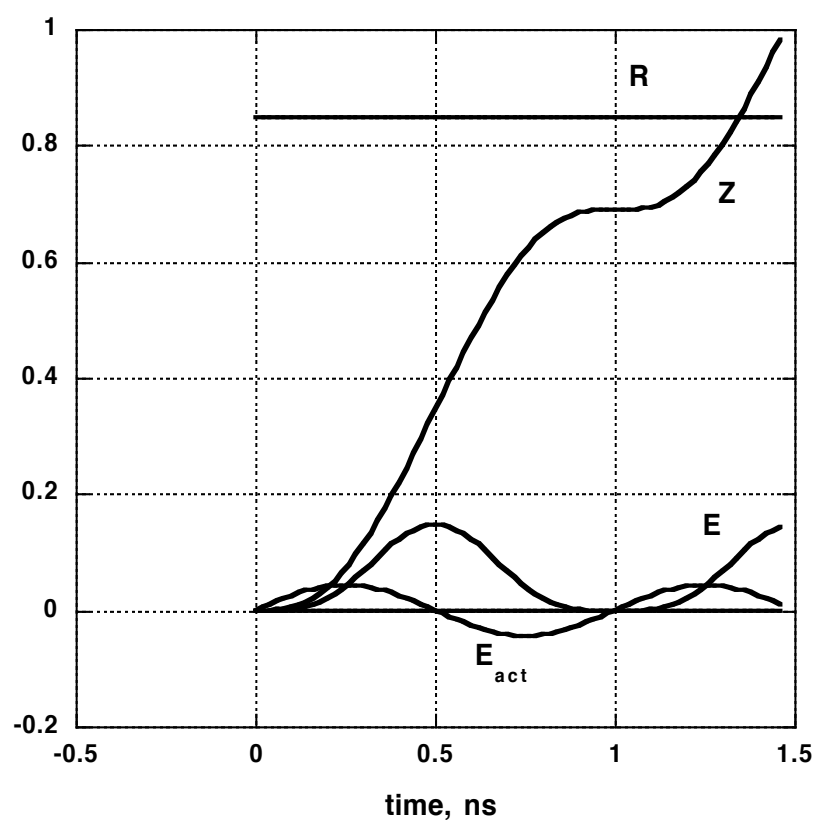

Fig. 21. Electron ejected near the wall at $\mathrm{R}_{0}=0.85$

\section{SUMMARY}

The above results and discussion are summarized as follows:

1. Accelerating gradient. Higher accelerating gradients are required for future demands like the TeV electron linear collider and muon-muon collider. A limit in the maximum electric field is imposed by the rf dark current and subsequent electrical breakdown.

2. Critical questions. What causes high dark current and breakdown? Can the breakdown threshold be increased? These questions have been subjected to experimental and theoretical investigations for a century, but the problems of $\mathrm{rf}$ dark current and breakdown remain unsolved today.

3. Breakdown problem. High-frequency rf breakdown at relatively low voltages is a single surface phenomenon. To match experimental data to theory, we used the field enhancement factor, which takes into account a localized field enhancement due to microprotrusions (surface irregularities) and absorbed gases. Surface processing decreases the field enhancement factor, but there are limits to the surface improvement following treatment. While the breakdown can be explained by electrode explosive emission, it is still necessary to find mechanisms related to high currents strong enough to form an ionized vapor cloud as the source of unlimited emission. 
4. Dark current problem. The solution of the dark current problem is still unclear. The dark current dependence on $\mathrm{E}_{0}$ has a threshold character. The field emission appears to be a multiplicative process. For such a multiplicative effect to exist $\square>1$. A considerable amount of experimental data and calculations exists for low-energy electron beams $(\mathrm{E}<1 \mathrm{MeV})$, but interactions at larger energies have not been well studied.

5. Breakdown analysis. We analyzed enhanced field emission due to separation of grains from a body by the electric field tension acting normal to the surface. The electric force, $\vec{F}_{E}=\frac{1}{2} \square \vec{E}=\frac{E^{2}}{8 \square} \vec{n}$ with maximal available electric field $E_{\max }=\sqrt{8 \square \square_{B}}>10 \frac{G e V}{m}$, is high enough to overcome the binding energy and the brittle destruction tension, $\square$ B. Thus, grain with size $a$ is separated from the body, and the separated grain produces enhanced field emission with $\mathrm{E}=\mathrm{E}_{\max }$. If the total current ispot determined by the Child-Langmuir law is large enough to vaporize this grain, breakdown can occur because the total current $\left(I=i_{\text {spot }} N_{\text {spot }}\right)$ is large enough to short circuit the electrodes. The derived criterion is

$$
E_{0}>\operatorname{lof} \square_{B}^{\square \frac{5}{14}}=\left.\frac{\square T_{i o n}}{\square}\right|^{\frac{4}{5}}
$$

where $\mathrm{f}$ is in $\mathrm{GHz}$, and $\mathrm{E}_{0}$ in $\mathrm{MeV} / \mathrm{m}$. Equation (4.17)

is close to the Kilpatrick criterion but depends on frequency. This criterion includes such electrode material properties as $\square, \square$, and $\square$ B. The Kilpatrick criterion also includes a parameter (but only one) depending of the electrode material.

6. Dark current analysis. The dark current significantly exceeds the Fowler-Nordheim current in enhancing the secondary electron emission transition due to relativistic and ultrarelativistic energies of ejected electrons accelerated in linacs. Such a transition does not occur because of simple energy increasing but because of relativistic electrons behaving qualitatively different from nonrelativistic electrons.

Other important conclusions from our work include:

1. The stopping power increases due to such relativistic effects as flattening of the electric field of an electron, which results in strong interactions with target electrons. Therefore, at ultrarelativistic energy $\left(\mathrm{E}>>\mathrm{mc}^{2}\right)$, the stopping power of electrons approached the stopping power of ions with the same energy but much less velocity. Ions with energy of more than $1 \mathrm{MeV} /$ nucleus have yield $\square>>1$. We anticipate that an electron with relativistic energy of $\mathrm{E}>5-10 \mathrm{MeV}$ also can produce $\square>1$.

2. The velocity limitation of the speed of light can result in easier satisfaction of synchronization.

3. Strong influence of the guide magnetic field results in a) more electrons reaching the opposite electrode due to straightening of the electron trajectory and b) more secondary electrons. 
The above findings are mostly qualitative, and the reported values should be regarded as having poor accuracy. To obtain reliable results, experimental and theoretical investigations are necessary.

\section{Theory:}

- Modeling of surface grain dynamics under high electric fields,

- Calculation of electric field near separating grains and current,

- Calculation of relativistic electron interactions with metals to find the secondary emission coefficient, and

- Modeling of an electron cloud of emitted and secondary electrons in linacs, taking into account its interaction with the metallic electrodes and walls.

\section{Experiment:}

- Breakdown dependence on material properties like conductivity, resistivity, grains size, and brittle destruction threshold,

- Secondary electron emission for high energy electrons, and

- Dependence of dark current on material properties and magnetic fields.

\section{ACKNOWLEDGEMENTS}

Argonne National Laboratory's work was supported by the U.S. Department of Energy, under contract W-31-109-Eng-38. 


\section{REFERENCES}

1. L. L. Laurent, "High Gradient rf Breakdown Studies," Ph.D. Thesis, Oregon Institute of Technology (2002).

2. Mesyats, Explosive Electron Emission, URO-Press, Ekaterinburg, p. 248 (1998).

3. J. G. Trump and R. T. Van de Graaf, "The Insulation of High Voltages in Vacuum," J. Appl. Phys., 18, 327 (1997).

4. Aarset, R. W. Cloud, and J. G. Trump, "Electron Emissions from Metals under High Energy Hydrogen Ion Bombardment,” J. Appl. Phys., 2511 (1954).

5. Bohne et al., "Sparking Measurements in a Single Gap Cavity," IEEE Trans. Nucl. Sci., 18, 568 (1971).

6. W. D. Kilpatrick, "Criterion for Vacuum Sparking Designed to Include both RF and DC,” Rev. Sci. Instr, 28, 824 (1957).

7. H. Matsumoto, "Dark Currents," $18^{\text {th }}$ Int. Linac Conf., Geneva, Switzerland, August 2630, 1996, KEK, preprint 96-120 (Sept. 1996).

8. R. V. Latham, High Voltage Vacuum Insulation, The Physical Basis, Academic Press, London, p. 245 (1981).

9. J. Norem et al., "Dark Current and X-ray Measurements of an $805 \mathrm{MHz}$ Pillbox Cavity," PAC-2003, Portland, OR, May 14-17, 2003, Abstract-TRAB003 (2003).

10. V. P. Kovalev, Secondary Electrons, Energoizdat, Moscow, p. 177 (in Russian) (1987). 


\section{Distribution List for ANL-ET-CPH-03/18}

$\underline{\text { Internal: }}$

J. P. Allain

J. N. Brooks

H. Drucker

R. Foote

D. Gruen
J. Harmon

A. Hassanein (10)

Z. Insepov

I. Konkashbaev (10)

V. Morozov
R. B. Poeppel

W. Shack

V. A. Sizyuk

V. I. Tolkach

R.A. Valentin

TIS Files

External:

DOE-SC (5)

ANL Libraries:

ANL-E

ANL-W

Main Library:

Brookhaven National Laboratory

Fermi National Accelerator Laboratory

Lawrence Berkeley National Laboratory

Lawrence Livermore National Laboratory

Los Alamos National Laboratory

Naval Research Laboratory

Sandia National Laboratories

Energy Technology Division Review Committee:

H.K. Birnbaum, University of Illinois at Urbana-Champaign

I. -W. Chen, University of Pennsylvania

F. P. Ford, Rexford, N.Y.

S.L. Rohde, University of Nebraska-Lincoln

H.S. Rosenbaum Fremont, CA

S.L. Sass, Cornell University

R. Zoughi, University of Missouri-Rolla 
External:

T. Bernat, Lawrence Livermore National Laboratory

G. Besenbruch, General Atomic

C. Bibeau, Lawrence Livermore National Laboratory

D. Colombant, Naval Research Laboratory

M. Friedman, Naval Research Laboratory

C. Halvorson, Schafer Laboratories

R. Hawryluk, Princeton Plasma Physics Laboratory

D. Haynes, Los Alamos National Laboratory

J. Hunn, Oak Ridge National Laboratory

Y. Kozaki, Osaka University, Japan

J. Lindl, Lawrence Livermore National Laboratory

Y. Martynenko, Kurchatov Institute, Russia

G. Nardella, U.S. Department of Energy

R. Schneider, U.S. Department of Energy

D. Schroen, Sandia National Laboratories

J. Swadener, Los Alamos National Laboratory

T. Norimatsu, Institute of Laser Engineering, Osaka University, Japan

M. Tillack, University of California, San Diego

Bibliothek, Max-Planck-Institute für Plasmaphysik, Germany

C.E.A. Library, Fontenay-aux-Roses, France

Librarian, Culham Laboratory, England

Thermonuclear Library, Japan Atomic Energy Research Institute, Japan

University of Wisconsin Library 